\title{
Teorema de Arrow-Sen e teoria da escolha fuzzy
}

\author{
Rafael Hernandez Jabur
}

\author{
DISSERTAÇÃO \\ $\mathrm{AO}$ \\ Instituto DE MATEmÁtica E EstatísticA \\ DA \\ Universidade DE SÃo Paulo \\ PARA \\ OBTENÇÃO DO TÍTULO \\ $\mathrm{DE}$ \\ Mestre em Matemática Aplicada
}

Programa: Matemática Aplicada

Orientador: Prof. Dr. Pedro Aladar Tonelli

Durante o desenvolvimento deste trabalho, o autor recebeu auxílio financeiro do CNPq

São Paulo, Junho de 2017 



\section{Teorema de Arrow-Sen e teoria da escolha fuzzy}

Esta é a versão original da dissertação elaborada pelo candidato Rafael Hernandez Jabur, tal como submetida à Comissão Julgadora. 



\section{Agradecimentos}

Agradeço a Deus, à minha família, aos amigos da vida, da matemática e da economia, ao meu orientador, e aos professores que tive a sorte de encontrar pelo caminho. Agradeço também ao IME-USP e ao CNPq pela bolsa concedida. 



\section{Resumo}

Racionalidade é um importante tema para a economia e qualquer outra área que se dedica à tomada de decisão. Ao longo do século XX, economistas deram diferentes definições do que seria um comportamento racional. Tais definições foram formalizadas matematicamente e, mediante uma hipótese plausível, mostradas equivalentes no teorema de ArrowSen. Após o surgimento da teoria de conjuntos fuzzy, diferentes autores se debruçaram na expansão através da matemática fuzzy de tais definições e equivalências. Este trabalho retoma o histórico dessas definições e a passagem da formalização clássica para a fuzzy. Também expõe resultados obtidos com provas alternativas e exemplos que mostram as limitações das equivalências numa abordagem sob a ótica da teoria de conjuntos fuzzy.

Palavras-chave: racionalidade, Arrow-Sen, fuzzy, função de escolha. 



\section{Abstract}

Rationality is among the most important subjects in economics and the study of decisionmaking process. Different definitions of what is a rational behavior were given in the $20^{\text {th }}$ century. The Arrow-Sen theorem shows that such definitions are equivalent under a plausible hypothesis. With the development of fuzzy theory, a fuzzy approach to this problem was given by different authors. This work shows this historical development of the definitions and the transition from the classical to the fuzzy view. It also presents the results obtained with alternative proofs and examples that show the limitations of the equivalences in the fuzzy setup.

Keywords: rationality, Arrow-Sen, fuzzy, choice function. 



\section{Sumário}

$1 \quad$ INTRODUÇÃO $\ldots \ldots \ldots \ldots \ldots \ldots \ldots$

2 HISTÓRICO DAS DEFINIÇÕES DE RACIONALIDADE ..... 2

3 TEORIA DA ESCOLHA CLÁSSICA E TEOREMA DE ARROW-SEN 4

$3.1 \quad$ Definições Preliminares $\ldots \ldots \ldots \ldots$

$3.2 \quad$ Formalização dos Conceitos de Racionalidade $\ldots \ldots \ldots$

$3.3 \quad$ Teorema de Arrow-Sen $\ldots \ldots \ldots \ldots$

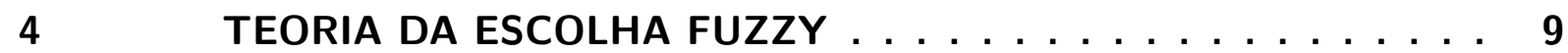

$4.1 \quad$ Fundamentos da Teoria de Conjuntos Fuzzy e Lógica Fuzzy . . . . . 9

4.2 Definições Preliminares da Teoria de Escolha Fuzzy $\ldots \ldots$. . . . 16

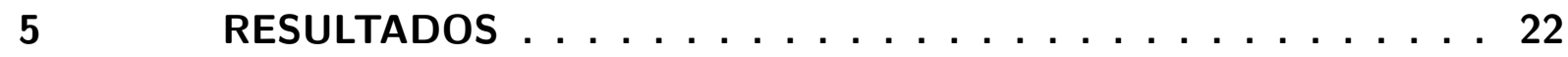

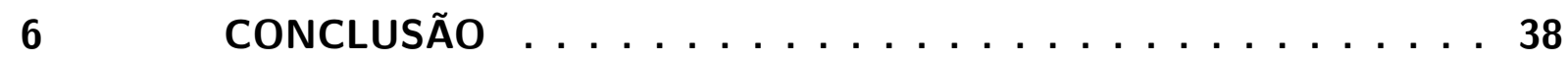

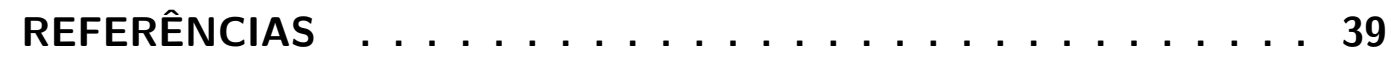

ANEXO A - NORMALIDADE DE UMA FUNÇÃO DE ESCOLHA 40

A.1 Normalidade na Teoria da Escolha Clássica . . . . . . . . . . 40

A.2 Normalidade na Teoria da Escolha Fuzzy . . . . . . . . . . . 41 



\section{Introdução}

O estudo sobre o comportamento do consumidor teve, no primeiro meado do século XX, uma mudança de tendência: da investigação do conceito de utilidade para a observação mais direta sobre as escolhas do consumidor. Isso fica explícito na seguinte passagem: "I propose, therefore, that we start anew in direct attack upon the problem, dropping off the last vestiges of the utility analysis" (SAMUELSON, 1938). Para tal, pressupostos sobre como agiria um consumidor racional surgiram ao longo do tempo. Ou seja, em última análise, definições sobre racionalidade. No decorrer dos anos, a noção de consumidor foi substituída por outra mais geral, a de uma função de escolha, e, no trabalho de Sen (1971), utilizando resultados de Arrow (1959), foi demonstrado que essas definições, sob uma hipótese bastante razoável, seriam equivalentes. Ou seja, que, se as ações de um agente que faz escolhas estivesse em acordo com alguma delas, também estaria conforme às outras. Essa equivalência é conhecida como teorema de Arrow-Sen.

Por outro lado, a partir de Zadeh (1965), foi sendo desenvolvida a teoria matemática fuzzy. Nela, não mais um elemento pertence ou não a um conjunto, mas possui um grau de pertencimento. Com isso, foram elaboradas ferramentas lógicas para lidar com proposições cujo valor de verdade não se restringe a verdadeiro, 1, ou falso, 0.

A partir de Orlovsky (1977), Banerjee (1995) e, posteriormente, de maneira mais sólida, em Georgescu (2007) e Martinetti et al. (2014), a teoria matemática fuzzy é incorporada à teoria da escolha - buscando responder, entre outras questões, se as equivalências descritas no teorema de Arrow-Sen continuam válidas ao se permitir que relações de preferência, espaços de escolha e funções de escolha sejam fuzzyficados.

Este trabalho busca compilar os resultados dessa investigação, bem como obter, em alguns casos, provas mais simples daquelas apresentadas pelos autores dos trabalhos citados. Para tal, primeiro traça, no Capítulo 2, um histórico das definições de racionalidade e a intuição por trás delas. Depois, no Capítulo 3, é apresentada a formalização matemática dessas noções e o teorema de Arrow-Sen. No Capítulo 4, são exibidas noções básicas da teoria matemática fuzzy e a passagem das definições do capítulo anterior para o arcabouço dos conjuntos fuzzy. O Capítulo 5 mostra os resultados e as limitações sobre as equivalências do teorema de Arrow-Sen quando tratadas no escopo da matemática fuzzy. Por fim, o Capítulo 6 concluí o trabalho e aponta possíveis direções a serem tomadas a partir dos resultados expostos. 


\section{Histórico das Definições de Racionalidade}

A primeira noção de racionalidade minimamente formalizada surge em Samuelson (1938). Nesse trabalho, o, autor introduz uma relação, a de preferência revelada 1 , da seguinte maneira: sendo $A$ e $B$ duas cestas distintas de bens, se é possível para o consumidor, mediante uma dada renda e um dado conjunto de preços dos bens, comprar a cesta $A$ ou comprar a cesta $B$ e ele opta por adquirir a cesta $A$, essa cesta é revelada preferida à cesta $B$. Tal relação seria assimétrica, ou seja, se $A$ é revelada preferida a $B, B$ não pode ser, para a mesma renda e mesmo conjunto de preços dos bens, revelada preferida a $A$. A partir dessa relação, o autor faz um postulado sobre o que consideraria

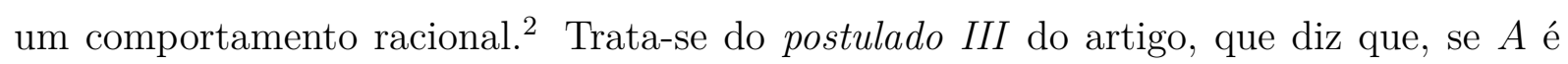
revelada preferida a $B$ para uma dada renda e um dado conjunto de preço dos bens, $B$ não pode ser revelada preferida a $A$ em qualquer outra situação de renda e conjunto de preços. Esse postulado recebe posteriormente, no trabalho de Sen (1971), o nome de axioma fraco de preferência revelada (WARP ${ }^{34}$ ).

Já em Houthakker (1950), aparece outra noção de racionalidade derivada da de Samuelson (1938). Segundo o autor, o postulado do WARP não excluiria a possibilidade de uma dada cesta $A$ ser revelada preferida a $B, B$ a $C$ e $C$ a $A$, sendo $A, B$ e $C$ diferentes - o que seria um "paradoxo" para um consumidor racional.5 Para contornar isso, o autor propõe um novo axioma que definiria um comportamento raciona] sequência finita de cestas de bens. Se, para todo $i=2,3, \ldots, n, A_{i}$ é revelada preferida a $A_{i-1}$ (para uma dada renda e conjunto de preços) ou é igual a $A_{i-1}$, e, para ao menos um $j \in\{2, \ldots, n\}, A_{j}$ é diferente de $A_{j-1}$, então $A_{1}$ não pode ser revelada preferida a $A_{n} \mathrm{em}$ qualquer situação de renda e conjunto de preços. Esse axioma, na nomenclatura de Sen (1971), é o axioma forte de preferência revelada (SARP 7 ).

Uma outra definição de racionalidade aparece pela primeira vez em Richter (1966). Trata-se da congruência de comportamento. Um consumidor seria congruente quando seu comportamento nunca se desviasse do seguinte: se uma cesta de bens $A$ foi revelada preferida a uma cesta $B$ e, dada uma situação de renda e preços, $A$ e $B$ estão disponíveis e $B$ está entre as cestas que o consumidor considera escolher, $A$ também deve estar. Caso

1 Embora essa terminologia tenha surgido posteriormente - não estando presente explicitamente em Samuelson (1938).

2 Para o autor, esse postulado seria satisfeito na quase totalidade dos comportamentos econômicos.

3 Do inglês Weak Axiom of Revealed Preference.

4 As siglas dos axiomas serão mantidas em inglês.

5 Essa afirmação parece ser inconsistente com os resultados que serão expostos adiante. No entanto, é preciso observar que o autor não pressupõe a hipótese que será utilizada.

6 Além de contornar o "paradoxo", o autor também prova que, usando o axioma e mais algumas hipóteses, é possível construir curvas de indiferença a partir da observação das escolhas de um consumidor e, assim, atribuir a ele uma função utilidade.

7 Do inglês Strong Axiom of Revealed Preference. 
essa preferência revelada tenha que ser direta $\left.\right|^{8}$ e a congruência é cumprida, é satisfeito, na nomenclatura de Sen (1971), o axioma fraco de congruência (WCA9?. Já, se é permitido que a preferência revelada seja indireta 10 é satisfeito o axioma forte de congruência $\left(\mathrm{SCA}^{11}\right)$.

Duas outras condições para um comportamento racional são a de contração e expansão. A condição de contração aparece em Chernoff (1954) e a de expansão em Sen (1969). A de contração, denominada condição $\alpha$, diz que, se um consumidor racional escolhe uma cesta de bens $A$ de um conjunto de cestas disponíveis, ele continuará escolhendo $A$ caso esse conjunto diminua, mas $A$ ainda esteja disponível. Já a condição de expansão, denominada condição $\beta$, diz que um consumidor racional que é indiferente entre duas cestas de um determinado conjunto continuará indiferente a elas caso novas cestas sejam acrescentadas nesse conjunto.

$8 A$ é diretamente revelada preferida a $B$ quando $A$ e $B$ estão disponíveis para escolha numa determinada situação de renda e preços e $A$ é escolhida.

9 Weak Congruence Axiom.

$10 A$ é indiretamente revelada preferida a $B$ quando existe um conjunto de cesta de bens $\left\{C_{1}, \ldots, C_{n}\right\}$ em que $A$ é diretamente revelada preferida a $C_{1}, C_{1}$ a $C_{2}, \ldots$, e $C_{n}$ a $B$.

11 Do inglês, Strong Congruence Axiom. 


\section{Teoria da Escolha Clássica e Teorema de Arrow-Sen}

Neste capítulo é apresentada, sob a ótica da teoria clássica de conjuntos, a definição formal de função de escolha, que é uma generalização da noção de consumidor, bem como a formalização dos axiomas e das condições de racionalidade presentes na seção anterior. Por fim, será exposto o Teorema de Arrow-Sen, que mostra que as caracterizações de racionalidade, sob certa hipótese, são, na verdade, equivalentes. As ideias e resultados deste capítulo estão presentes em Sen (1971).

\subsection{Definições Preliminares}

Seja $X$ um conjunto de alternativas. Por exemplo, num caso concreto econômico em que se tenha $n$ bens, cada ponto em $X=\mathbb{R}_{+}^{n}$ representa uma cesta de bens. 1. Seja $\mathcal{B}$ uma família não-vazia de conjuntos $\emptyset \neq S \subseteq X$. Voltando ao caso econômico, cada $S \in \mathcal{B}$ representa um conjunto de cestas de bens. É possível definir $\mathcal{B}$ de maneira que cada $S \in \mathcal{B}$ caracterize uma restrição orçamentária. Isso é feito da seguinte maneira: dado um vetor de preços $\left(p_{1}, \ldots, p_{n}\right)^{2}$ e uma renda $r$, somente as cestas $\left(x_{1}, \ldots, x_{n}\right)$ que cumprem $x_{1} p_{1}+\ldots+x_{n} p_{n} \leq r$ estão disponíveis para a compra. Nesse caso, cada $S_{(p, r)} \in \mathcal{B}$ pode ser identificado com um vetor de preços $p$ e uma renda $r$. Ou seja, $x \in S_{(p, r)}$ quando $x$ está disponível para compra dado o vetor de preços $p$ e a renda $r$. O par $(X, \mathcal{B})$ é denominado espaço de escolha.

Definição 3.1. Dado um espaço de escolha $(X, \mathcal{B})$, uma função de escolha $C$ é um mapa $C: \mathcal{B} \rightarrow \mathcal{P}(X)$, onde $\mathcal{P}(X)$ é o conjunto de partes de $X$, tal que, para qualquer $S \in \mathcal{B}$, $\emptyset \neq C(S) \subseteq S$.

Interpretando economicamente a definição 3.1, $C$ representa um consumidor que se depara com uma restrição orçamentária, dada por um vetor de preços $p$ e uma renda $r$, que leva ao conjunto de cesta de bens $S_{(p, r)}$ estar disponível para compra. Esse consumidor deve fazer uma escolha entre as cestas disponíveis, isto é, as cestas que pertencem a $S_{(p, r)}$. O consumidor deve escolher alguma coisa, ou seja, $C\left(S_{(p, r)}\right) \neq \emptyset$. Além disso, sua escolha deve estar entre as cestas que podem ser adquiridas, ou seja, $C\left(S_{(p, r)}\right) \subseteq S_{(p, r)}$.

Outra coisa a se notar é que, na definição 3.1, dado um $S \in \mathcal{B}, C(S)$ pode conter mais de um elemento. Nada se perde com isso, uma vez que os resultados válidos para essa definição também valem quando, para todo $S \in \mathcal{B}, C(S)$ contém apenas um elemento.

1 Cada coordenada $i ; i=1, \ldots, n$; representa a quantidade do bem $i$ na cesta.

$2 p_{i} ; i=1, \ldots, n$; o preço do bem $i$. 
Além disso, na teoria econômica, existem situações de indiferença do consumidor entre cestas de bens, o que não seria contemplado se na definição não fosse permitido, dado $S \in \mathcal{B}, C(S)$ conter mais de um elemento. Por exemplo, se o consumidor é indiferente entre as cestas $x$ e $y$, e $S=\{x, y\}$, faz sentido $C(S)=\{x, y\}$.

Uma relação binárid em $X$ é uma função $Q: X^{2} \rightarrow\{0,1\} . Q(x, y)=1$ designa que $x$ está relacionado a $y$ no escopo de $Q$, enquanto $Q(x, y)=0$ que não está. Dadas duas relações, $Q$ e $R$, em $X$, é escrito $Q \subseteq R$ quando $Q(x, y) \leq R(x, y)$ para todo $x, y \in X$. É usual escrever $x Q y$ para indicar que $Q(x, y)=1$ e $\neg(x Q y)$ para indicar que $Q(x, y)=0$. As seguintes propriedades podem estar presentes numa relação $Q$ em $X$ :

- Transitividade: para quaisquer $x, y, z \in X$, se $x Q z$ e $z Q y$, então $x Q y$;

- Reflexividade: para qualquer $x \in X, x Q x$;

- Totalidade: para quaisquer $x, y \in X$ distintos, $x Q y$ ou $y Q x$;

- Completude: para quaisquer $x, y \in X, x Q y$ ou $y Q x$;

- Regularidade: $Q$ é completa e transitiva.

Definição 3.2. Dada uma relação $Q$ em $X$, seu fecho transitivo é a relação $Q^{*}$ que cumpre as seguintes propriedades:

1. $Q^{*}$ é reflexiva.

2. $Q \subseteq Q^{*}$.

3. Se uma relação $R$ em $X$ é tal que $R$ é transitiva e $Q \subseteq R$, então $Q^{*} \subseteq R$.

Dado um espaço de escolha $(X, \mathcal{B})$ e uma função de escolha $C$, as relações em $X$ dadas na definição abaixo são denominadas relações de preferência revelada. Essas relações serão usadas na formalização das caracterizações de racionalidade.

Definição 3.3. Definidos um espaço de escolha $(X, \mathcal{B})$ e uma função de escolha $C$,

- $x R y$ quando, para algum $S \in \mathcal{B}, x \in C(S)$ e $y \in S$.

- $x P y$ quando $x R y$ e $\neg(y R x)$.

- $x \widetilde{P} y$ quando, para algum $S \in \mathcal{B}, x \in C(S)$ e $y \in S \backslash C(S)$.

- $W$ é o fecho transitivo de $R$.

- $P^{*}$ é o fecho transitivo de $\widetilde{P}$.

3 A partir de agora apenas relação.

4 No contexto econômico, dizer que $Q$ é uma relação de preferência de um consumidor sobre um conjunto de cestas de bens, significa que, se $x Q y$, o consumidor tem para si que a cesta de bens $x$ é tão boa quanto a cesta de bens $y$. 
Se $\mathcal{B}$ contém todos os pares $\{x, y\} \subseteq X$, também pode ser definida:

- $x \bar{R} y$ quando $x \in C(\{x, y\})$.

No caso de um consumidor, $x R y$ indica que a cesta de bens $x$ foi escolhida quando a cesta $y$ também estava disponível; $x P y$ significa que $x$ foi escolhida quando $y$ estava disponível e que $y$ nunca foi escolhida quando $x$ estava disponível; e $x \widetilde{P} y$ representa que $x$ foi escolhida e $y$ rejeitada quando ambas estavam disponíveis. Já a relação $x W y$ $\left(x P^{*} y\right)$ indica que existe um conjunto de cestas $\left\{z_{1}, \ldots, z_{n}\right\}$ tal que $x$ foi escolhida (e $z_{1}$ rejeitada) quando $z_{1}$ estava disponível, $z_{1}$ foi escolhida (e $z_{2}$ rejeitada) quando $z_{2}$ estava disponível,..., e $z_{n}$ foi escolhida (e $y$ rejeitada) quando $y$ estava disponível.

Definição 3.4. Dado um espaço de escolha $(X, \mathcal{B})$ e uma função de escolha $C$, a imagem no sentido de Sen ${ }^{5}$ de $C$ é o mapa $\widehat{C}: \mathcal{B} \rightarrow \mathcal{P}(X)$ definido por $\widehat{C}(S)=\{x \in S \mid x R y, \forall y \in$ $S\}$.

Ou seja, se $S$ representa um conjunto de cesta de bens e $C$ um consumidor, $\widehat{C}$ é o conjunto de cestas que o consumidor revelou serem preferidas a todas as cestas de $S$. Note que, se $x \in C(S), x R y$ para todo $y$ em $S$. Logo, $C \subseteq \widehat{C}$ e, além disso, $\widehat{C} \subseteq S$. Portanto, $\widehat{C}$ é também uma função de escolha. ${ }^{6}$

Definição 3.5. Dado um espaço de escolha $(X, \mathcal{B})$ e uma função de escolha $C, C$ é normal quando $C=\widehat{C}]^{7}$

Essencialmente, do ponto de vista econômico, a função de escolha que representa um consumidor é dita normal se, dado um conjunto de cestas de bens, ele não deixará uma cesta que foi relevada ser ao menos tão boa para ele quanto as outras do conjunto fora do escopo de escolha.

\subsection{Formalização dos Conceitos de Racionalidade}

Abaixo estão formalizados os conceitos de racionalidade expostos na seção 2, tal como feito em Sen (1971).

Seja um espaço de escolha $(X, \mathcal{B})$ e uma função de escolha $C$. $C$ cumpre (ou satisfaz)

- WARP, quando para todos $x, y \in X, x \widetilde{P} y$ implica $\neg(y R x)$.

- SARP, quando para todos $x, y \in X, x P^{*} y$ implica $\neg(y R x)$.

- WCA, quando para todo $S \in \mathcal{B}$ e todos $x, y \in X ; x R y, y \in C(S)$ e $x \in S$ implica $x \in C(S)$.

5 Daqui adiante apenas imagem.

6 Note que a relação $R$ é definida a partir de $C$.

7 Uma discussão mais aprofundada sobre normalidade encontra-se no anexo $\mathrm{A}$ Esta definição é a que se encontra em Sen (1971). 
- SCA, quando para todo $S \in \mathcal{B}$ e todos $x, y \in X ; x W y, y \in C(S)$ e $x \in S$ implica $x \in C(S)$.

- condição $\alpha$, quando para todos $S_{1}, S_{2} \in \mathcal{B}$ e todo $x \in X ; S_{1} \subseteq S_{2}, x \in C\left(S_{2}\right)$ e $x \in S_{1}$ implica $x \in C\left(S_{1}\right)$. $^{8}$

- condição $\beta$, quando para todos $S_{1}, S_{2} \in \mathcal{B}$ e todos $x, y \in X ; S_{1} \subseteq S_{2}, x, y \in C\left(S_{1}\right)$ implica que $x \in C\left(S_{2}\right)$ se, e somente se, $y \in C\left(S_{2}\right)$.

\subsection{Teorema de Arrow-Sen}

O teorema exposto neste seção é uma compilação dos resultados presentes em Arrow (1959), Sen (1971) e Sen (1977). Como fruto desses trabalhos, sabe-se que as definições de racionalidade expostas na seção 2 e formalizadas na seção 3.2 são, na presença da hipótese apresentada a seguir, equivalentes. O resultado, porém, não se resume a isso. Também é demonstrado que, se há racionalidade no comportamento de escolha, existe, então, uma regularidade na preferência revelada por essa escolha. Ou seja, essa preferência revelada cumpre as propriedades de completude e transitividade, o que é esperado de um comportamento racional.

Considere um espaço de escolha $(X, \mathcal{B})$. Ele pode cumprir o seguinte:

Hipótese $H: \mathcal{B}$ contém todos os conjuntos $\{\mathrm{x}\},\{\mathrm{x}, \mathrm{y}\}$ e $\{\mathrm{x}, \mathrm{y}, \mathrm{z}\}$, com $x, y, z \in X .^{9}$

Essa hipótese é fundamental para a validade do teorema. Porém, como discutido em Sen (1971), questões quanto a verificação dela para o caso de um consumidor real podem ser levantadas. Isso porque um espaço de escolha onde $\mathcal{B}$ é uma família de restrições orçamentárias não a cumpre. No entanto, o próprio autor responde a isso. Primeiro, uma teoria da escolha não se restringe ao caso do consumidor, tendo seus resultados válidos para outros campos, como, por exemplo, numa escolha política em que se deve votar a partir de um conjunto de candidatos possíveis. Mas, além disso, mesmo na situação de um consumidor cujo espaço de escolha num caso concreto não cumpra $H$, a interpretação sobre as escolhas de um consumidor vai além das escolhas diretamente observadas. Isso significa que, mesmo não se concretizando uma situação em que o consumidor tenha que escolher entre um par de cestas, é possível inferir qual seria a escolha, caso as escolhas observadas se conformem com uma definição de racionalidade. O caso que seria contrário a isso é aquele no qual se observa a conformidade com uma das definições de racionalidade, por exemplo, o WCA, mas se infere que o WCA não seria observado no caso hipotético

8 É de se notar que isso configura uma noção de independência entre qualquer $x \in C(S)$ e qualquer $y \in S \backslash C(S)$, uma vez que a presença ou não de $y$ em $S$ não mudará o fato de $x$ ser escolhido. Esse tópico, porém, não será discutido neste trabalho.

9 A hipótese original apresentada em Sen (1971) é a de que $\mathcal{B}$ contém todos subconjuntos finitos de $X$. Porém, como o próprio autor menciona no texto, essa hipótese original pode ser relaxada para a hipótese aqui exposta sem prejuízo para os resultados. 
desse mesmo consumidor se deparar com a opção de três ou duas cestas. E não há porque a priori achar que esse é o caso, mas, ao contrário, parece ser muito mais natural assumir que o consumidor que é consistente em suas escolhas observadas seja também consistente em escolhas hipotéticas.

Teorema 3.1. Seja um espaço de escolha $(X, \mathcal{B})$ que cumpra $H$ e $C$ uma função de escolha. São equivalentes:

(i) Cé normal e $R$ é regular;

(ii) WARP é satisfeito por $C$;

(iii) SARP é satisfeito por $C$;

(iv) WCA é satisfeito por $C$;

(v) SCA é satisfeito por $C$;

(vi) condição $\alpha$ e condição $\beta$ são satisfeitas por $C$.

A relevância desse teorema é patente, uma vez que sinaliza a natureza do conceito de racionalidade. Conseguir demonstrar que, mediante uma hipótese aceitável, definições de racionalidade que foram dadas por diversos autores são equivalentes e, além disso, que há uma correspondência entre a ação observada de um agente racional e propriedades de uma relação de preferência intrínseca a esse agente, expande a fronteira da discussão sobre racionalidade. Buscar um resultado similar no contexto da teoria de conjuntos fuzzy parece ser apropriado - e é o que será discutido nos capítulos seguintes. 


\section{Teoria da Escolha Fuzzy}

Neste capítulo são apresentadas noções básicas da teoria de conjuntos fuzzy, lógica fuzzy, e conceitos e ferramentas que serão utilizados para estender as definições dadas no capítulo anterior.

\subsection{Fundamentos da Teoria de Conjuntos Fuzzy e Lógica Fuzzy}

Schmitz (1999) descreve uma proposição para a lógica aristotélica como sendo uma sentença declarativa, ou seja, capaz de ser verdadeira ou falsa.1. Por exemplo, a frase "a maioria dos eleitores escolheu o candidato A" é uma proposição nesse sentido. Note que, assim sendo, para que uma sentença seja proposição, não é necessário que sua veracidade seja verificável concretamente.

Por sua vez, o conceito de conjunto segundo Georg Cantor (1845-1918) é "qualquer coleção de objetos, bem-definidos e diferenciáveis entre si, presentes em nossa intuição ou intelecto, que possa ser concebida como um todo. Esses objetos são chamados elementos ou membros do conjunto."(STOLL, 1979, p. 2, grifo do autor, tradução nossa). Segundo o autor, a passagem de objetos individuais para coleções de objetos concebidas como um todo está presente em nossa linguagem - como, por exemplo, qualquer substantivo coletivo: assembleia, legião, rebanho. Podemos assim definir um conjunto $S$ como o "conjunto de livros", onde "livro" é qualquer escrito impresso que contenha mais de um milhão de caracteres. Nesse caso, a sentença " $x$ é um livro" ou " $x \in S$ " é uma proposição, pois é capaz de ser classificada como verdadeira ou falsa.

No entanto, como discutido em Zadeh (1965), certas classes de objetos encontrados no mundo real não têm um critério bem-definido para determinar a pertinência ou não de um dado objeto a essa classę Voltando ao exemplo de $S$ ser o conjunto de livros, o critério dado "qualquer escrito impresso que contenha mais de um milhão de caracteres" parece artificial. Se $x$ é um impresso escrito com um milhão de caracteres, $x$ não é um livro, $x \notin S$, enquanto $y$ um impresso escrito com um milhão e um caracteres é, $y \in S$. Isso soa contra-intuitivo e essa impressão vem do fato de que a sentença " $x \in S$ ", para certas coleções de objetos $S$ presentes na nossa linguagem habitual, não se comporta como uma proposição aristotélica - no sentido de que há uma inerente inexatidão ou vagueza sobre sua condição de verdade. Para lidar com essa imprecisão, o autor sugere a introdução do conceito de conjunto fuzzy.

1 Uma discussão sobre o que é necessário para que algo seja uma proposição, ou seja, dotado da propriedade de ser falso ou verdadeiro, foge do propósito deste trabalho.

2 Usualmente, na teoria de conjuntos fuzzy, diz-se pertinência ao invés de pertencimento. 
Definição 4.1. Dado um conjunto universo $X$, um conjunto fuzzy $F$ de $X$ é uma função $F: X \rightarrow[0,1]$. Para todo $x \in X, F(x)$ é o grau de pertinência de $x$ ao conjunto $F:^{3}$

Um conjunto fuzzy $F$ de $X$ é não-zero quando $F(x)>0$ para ao menos um $x \in X$. Além disso, $F$ é normal quando $F(x)=1$ para ao menos um $x \in X$. Denomina-se $\mathcal{F}(X)$ a família de todos os conjuntos fuzzy não-zeros de $X$. Dados dois conjuntos fuzzy $F$ e $G$, $F \subseteq G$ quando $F(x) \leq G(x)$, para todo $x \in X$. Designa-se $\left[x_{1}, \ldots, x_{n}\right]$ o conjunto fuzzy $F$ tal que $F(x)=1$ quando $x \in\left\{x_{1}, \ldots, x_{n}\right\}$ e, caso contrário, $F(x)=0$. Um conjunto fuzzy $F$ tal que $F(x)=1$ ou $F(x)=0$ para qualquer $x \in X$ é denominado um conjunto crisp.

Mas a vagueza sobre a condição de verdade não recaí somente em proposições do tipo $x \in S$. Na lógica fuzzy, o valor de verdade de qualquer proposição pertence ao conjunto $[0,1]]^{4}$ Assim sendo, surge a questão sobre como tratar os conectivos lógicos, uma vez que o cálculo proposicional clássico só lida com valores de verdade pertencentes a $\{0,1\}$.

Por exemplo, considere o conetivo lógico "e" [5 Dadas " $a$ " e " $b$ " duas proposições, na lógica clássica, a proposição " $a$ e $b$ " é verdadeira somente quando ambas " $a$ " e " $b$ " o são. Assim sendo, temos a seguinte tabela de verdade (o conectivo "e" representado por $*$ ):

\begin{tabular}{|c|c|c|}
\hline$a$ & $b$ & $a * b$ \\
\hline 1 & 1 & 1 \\
\hline 1 & 0 & 0 \\
\hline 0 & 1 & 0 \\
\hline 0 & 0 & 0 \\
\hline
\end{tabular}

Ou seja, o conectivo lógico "e" funciona como um operador $\{0,1\}^{2} \rightarrow\{0,1\}$, satisfazendo as propriedades de: 1 como identidade, comutatividade, associatividade e crescente em cada argumento. Portanto, ao expandir o conectivo "e" à perspectiva não-binária da lógica fuzzy, além de estender o domínio e o contradomínio do operador para $[0,1]^{2}$ e $[0,1]$ respectivamente, é preciso considerar essas propriedades como necessárias. Operadores com tais propriedades, segundo Nguyen e Walker (1997), já apareceram na teoria de espaços métricos probabilístico e são denominados normas triangulares.

Definição 4.2. Uma t-norma (norma triangular) é uma operação binária $*:[0,1]^{2} \rightarrow$ $[0,1]$ que satisfaz as seguintes propriedades para quaisquer $x, y, z \in[0,1]$ :

(i) $1 * x=x$;

(ii) $x * y=y * x$;

3 Segundo Zadeh (1965), o intervalo [0,1] poderia ser substituído por um conjunto parcialmente ordenado. Porém, a escolha do intervalo soa mais intuitiva, representando um continuum de valores de verdade.

4 O que foi dito na nota 3 vale também para esse caso mais geral.

5 Os conceitos colocados a seguir foram retirados de Nguyen e Walker (1997) e Georgescu (2007). 
(iii) $x *(y * z)=(x * y) * z$;

(iv) se $y \leq z, x * y \leq x * z$.

Note que, para qualquer $x \in[0,1], 0 * x \leq 0 * 1=0$, a desigualdade por $x \leq 1$ e a igualdade por 1 como identidade. Pela propriedade de comutatividade, também $x * 0=0$. Além disso, uma t-norma é dita contínua pela esquerda (direita) quando é um operador binário contínuo pela esquerda (direita) e contínua quando é contínua pela esquerda e pela direita.

Abaixo seguem alguns exemplos de t-norma $(\wedge$ representando o operador binário de mínimo e $\vee$ o de máximo):

- T-norma Drástica ${ }^{6}: x *_{D} y=\left\{\begin{array}{ll}x \wedge y, & \text { se } x \vee y=1 \\ 0, & \text { caso contrário }\end{array}\right.$;

- T-norma Produto: $x *_{P} y=x y$;

- T-norma de Gödel: $x *_{G} y=x \wedge y$;

- T-norma de Eukasiewicz: $x *_{L} y=0 \vee(x+y-1)$.

Similarmente, para o conectivo lógico "implica", obtemos, para valores verdade em $\{0,1\}$, a seguinte tabela ("implica" sendo representado por $\Longrightarrow$ ):

\begin{tabular}{|c|c|c|}
\hline$a$ & $b$ & $a \Longrightarrow b$ \\
\hline 1 & 1 & 1 \\
\hline 1 & 0 & 0 \\
\hline 0 & 1 & 1 \\
\hline 0 & 0 & 1 \\
\hline
\end{tabular}

Da mesma maneira que no caso anterior, um operador que represente tal conectivo no contexto fuzzy deve ter seu domínio e contradomínio estendidos para abarcar as possibilidades de valores verdade entre $[0,1]$ e, além disso, ser conforme à tabela acima quando restrito aos valores $\{0,1\}$.

Como esperado, há mais de uma maneira de se construir tais operadores. No entanto, este trabalho utilizará como operador para "implicação fuzzy" o resíduo associado a uma t-norma, definido abaixo, com a justificativa para essa escolha a seguir.

Definição 4.3. O resíduo associado a uma t-norma * é a operação binária $\longrightarrow{ }_{*}:[0,1]^{2} \rightarrow$ $[0,1]$ dada por:

$$
x \longrightarrow * y=\bigvee_{c \in[0,1]}[x * c \leq y]
$$

onde $\bigvee$ é o supremo.7

\footnotetext{
6 Um exemplo de t-norma que não é contínua.

7 No caso da t-norma estar subentendida, escreve-se apenas $\longrightarrow$ ao invés de $\longrightarrow$ *
} 
Por um lado, é assumido que vale a regra de inferência modus ponens. Assim sendo, considerando as proposições " $a$ ", " $b$ " e " $c$ " $\equiv$ " $a \Longrightarrow b$ ", caso " $a$ " e "c" sejam verdadeiras, via modus ponens, "b" também o é. Expandindo para uma perspectiva fuzzy, pode-se dizer que " $b$ " é sempre ao menos tão verdadeiro quanto a proposição " $a$ e $c$ ". Logo, o valor verdade de " $c$ " deve ser tal que valha $b \geq a * c$ (sem aspas para o valor verdade da proposição). Por outro lado, os valores de " $a \Longrightarrow b$ " da tabela de verdade para os casos da lógica binária são os mesmos dados por $\bigvee_{c \in[0,1]}\{a * c \leq b\}$, para qualquer t-norma $*$. Além disso, a implicação definida dessa forma é monotônica no seguinte sentido: se "a" implica "b", então "a e c" também implica "b". Isso pode ser visto com a proposição a seguir ${ }^{8}$

Proposição 4.1. Sejam $a, b, c \in[0,1]$ e uma t-norma $*$. Então, $a \longrightarrow b \leq(a * c) \longrightarrow b$.

Demonstração. Considere $C_{1}=\{x \in[0,1] \mid x * a \leq b\}$ e $C_{2}=\{x \in[0,1] \mid x * a * c \leq b\}$. Seja $y \in C_{1}$. Então, $y * a * c \leq y * a \leq b$. Logo, $y \in C_{2}$. Assim sendo, $C_{1} \subseteq C_{2}$. Portanto, $\sup C_{1} \leq \sup C_{2}$.

Para qualquer t-norma, $x \leq y$, se, e somente se, $a \longrightarrow b=1$. Quando $x>y$, são obtidos os seguintes resíduos associados a cada t-norma citada como exemplo:

$$
\begin{aligned}
& \text { - } x \longrightarrow_{*_{D}} y=\left\{\begin{array}{ll}
1, & \text { se } x<1 \\
y, & \text { se } x=1
\end{array} ;\right. \\
& \text { - } x \longrightarrow_{*_{P}} y=\frac{y}{x} ; \\
& \text { - } x \longrightarrow_{*_{G}} y=y ; \\
& \text { - } x \longrightarrow_{*_{L}} y=1-y+x .
\end{aligned}
$$

Seguindo a mesma linha de raciocínio, na expansão do operador referente ao conectivo lógico "equivale a" (ou "se, e somente se"), este trabalho utiliza o biresíduo, definido a seguir.

Definição 4.4. O biresíduo associado a uma t-norma $*$ é a operação binária $\rho_{*}:[0,1]^{2} \rightarrow$ $[0,1]$ dada por:

$$
\rho_{*}(x, y)=(x \longrightarrow * y) *(y \longrightarrow * x)^{9}
$$

Segundo Stoll (1979), uma proposição modificada pela palavra "não" é uma negação. Por exemplo, "107 não é um número primo" é a negação de "107 é um número primo". Em termos de linguagem usual, sendo " $a$ " uma proposição, podemos escrever a negação de " $a$ " como"não é o caso que $a$ ". De acordo com o valor verdade de " $a$ ", é obtida a seguinte tabela de verdade ( $\neg$ representando "não é o caso que"):

8 Para uma discussão mais ampla sobre implicações, ver capítulo 4 de Barros, Bassanezi e Lodwick (2017), especialmente seção 4.4 .

9 No caso da t-norma estar subentendida, escreve-se apenas $\rho$ ao invés de $\rho_{*}$ 


\begin{tabular}{|c|c|}
\hline$a$ & $\neg a$ \\
\hline 1 & 0 \\
\hline 0 & 1 \\
\hline
\end{tabular}

Da tabela, é retirada a primeira propriedade necessária ao expandir a negação para um contexto fuzzy, qual seja, uma negação fuzzy $\eta$ deve cumprir $\eta(1)=0$ e $\eta(0)=1$. Mas, além disso, é intuitivo pensar que, dadas duas proposições " $a$ " e " $b$ ", se o valor verdade de " $a$ " for maior que o valor de verdade de " $b$ ", $\eta(a) \leq \eta(b)$ - ou seja, a negação deve ser não-crescente.

Como no caso da implicação, há mais de uma forma de se obter as propriedades do parágrafo anterior. Por exemplo, o operador $\eta(x)=1-x$ as cumpre. Mas, mantendo a linha de Georgescu (2007), este trabalho utiliza a definição a seguir.

Definição 4.5. A negação associada a uma t-norma * é uma operação unária $\neg_{*}:[0,1] \rightarrow$ $[0,1]$ dada por:

$$
\neg_{*} x=x \longrightarrow_{*} 0=\bigvee_{c \in[0,1]}[x * c=0]
$$

No caso da t-norma estar subentendida, escreve-se apenas $\neg$ ao invés de $\neg$ *

Proposição 4.2. O operador definido em 4.5 cumpre as seguintes propriedades para qualquer t-norma:

(i) $\neg 1=0$ e $\neg 0=1$;

(ii) $x \leq y \Longrightarrow \neg y \leq \neg x$.

Demonstração. (i) Como $1 * c=c$ para qualquer $c \in[0,1]$, se $c>0$, então $1 * c>0$. Por outro lado, $1 * 0=0$;

(ii) como $x \leq y$, para todo $c \in[0,1]$, se $c * y=0$, então $c * x=0$. Assim sendo, $C_{y} \subseteq C_{x}$, onde $C_{x}=\{c \in[0,1] \mid c * x=0\}$ e $C_{y}$ definido analogamente. Logo, $\sup C_{y} \leq \sup C_{x}$.

Há ainda uma terceira propriedade, que é a de que o valor verdade da negação da negação de uma proposição é o mesmo que o valor de verdade da proposição - ou seja, que vale $\eta(\eta(a))=a$. Quando essa propriedade, em conjunto com as outras duas, é satisfeita por um operador, tal operador é denominado negação forte. Além disso, se $\neg_{*}\left(\neg_{*} x\right)=x$, diz-se que a t-norma $*$ gera uma negação forte. Associadas a cada t-norma dada como exemplo, são geradas as seguintes negações:

$$
\text { - } \neg *_{*_{D}} x= \begin{cases}1, & \text { se } x<1 \\ 0, & \text { se } x=1\end{cases}
$$


- $\neg_{*_{P}} x=\left\{\begin{array}{ll}1, & \text { se } x=0 \\ 0, & \text { se } x>0\end{array} ;\right.$

- $\neg_{*_{G}} x=\left\{\begin{array}{ll}1, & \text { se } x=0 \\ 0, & \text { se } x>0\end{array}\right.$;

- $\neg_{*_{L}} x=1-x$.

Note que, dos exemplos mencionados, apenas a t-norma de Łukasiewicz gera uma negação forte.

A proposição a seguir trata de propriedades envolvendo os operadores lógicos fuzzy definidos acima que serão utilizadas em demonstrações na seção 5 .

Proposição 4.3. Para quaisquer $x, y \in[0,1]$, e * uma t-norma contínua pela esquerda, vale que:

(i) $x *(x \longrightarrow y) \leq y$;

(ii) $x * \neg x=0$;

(iii) $x * y=0 \Longleftrightarrow x \leq \neg y$;

(iv) $x \leq \neg \neg x$.

Demonstração. (i) Como a t-norma $*$ é contínua pela esquerda, $x \longrightarrow y \in\{c \in[0,1] \mid$ $c * x \leq y\}$. Logo, $(x \longrightarrow y) * x \leq y$;

(ii) $x * \neg x=x *(x \longrightarrow 0) \leq 0$;

(iii) se $x * y=0$, então $x \leq \bigvee_{c \in[0,1]}\{y * c=0\}=\neg y$. Por outro lado, se $x \leq \neg y$, então $x * y \leq \neg y * y=0$;

(iv) como $x * \neg x=0, x \leq \bigvee_{c \in[0,1]}\{c * \neg x=0\}=\neg \neg x$.

Outro conceito importante para este trabalho é o de relações, que assim como os outros, pode ser expandido dentro de uma perspectiva fuzzy. Ou seja, o valor verdade da proposição " $x R y$ " pode ser tomado em qualquer número real do intervalo $[0,1]$ - chegando à definição a seguir.

Definição 4.6. Uma relação binária fuzzy ${ }^{10} Q$, é uma operação binária $Q: X^{2} \rightarrow[0,1]$. Para todo $(x, y) \in X^{2}, Q(x, y)$ é o grau da relação $Q$ entre $x$ e $y$.

10 Daqui adiante, apenas relação fuzzy. 
Sendo a relação fuzzy uma generalização do conceito de relação, é de se esperar que as propriedades que podem incidir sobre uma relação sejam estendidas para o caso fuzzy. Isso pode ser feito de mais de uma maneira - o exemplo mais claro sendo o da definição de transitividade. Porém, este trabalho irá se ater às definições usadas em Georgescu (2007).

Dada uma t-norma *, uma relação fuzzy $Q$ em $X$ pode apresentar as seguintes propriedades:

- *-transitividade: $Q(x, z) * Q(z, y) \leq Q(x, y)$, para todos $x, y, z \in X$;

- *-quasi-transitividade: $P_{Q}(x, z) * P_{Q}(z, y) \leq P_{Q}(x, y)$, para todos $x, y, z \in X$, onde $P_{Q}(x, y)=Q(x, y) * \neg Q(y, x)$

- totalidade: $Q(x, y)>0$ ou $Q(y, x)>0$, para $x, y \in X$ distintos;

- totalidade forte: $Q(x, y)=1$ ou $Q(y, x)=1$, para $x, y \in X$ distintos;

- completude: $Q(x, y)>0$ ou $Q(y, x)>0$, para todos $x, y \in X$;

- completude forte: $Q(x, y)=1$ ou $Q(y, x)=1$, para todos $x, y \in X$;

- reflexividade: $Q(x, x)>0$, para todo $x \in X$;

- reflexividade forte: $Q(x, x)=1$, para todo $x \in X$;

- regularidade: $Q$ é fortemente completa e $*$-transitiva.

Será escrito transitividade e quasi-transitividade ao invés de $*$-transitividade e $*$-quasitransitividade quando a t-norma envolvida estiver subentendida.

Note que, se uma relação fuzzy $Q$ é fortemente completa, $\neg Q(x, y) \leq Q(y, x)$, pois, se $Q(y, x)<1$, então $Q(x, y)=1$ e, portanto, $\neg Q(x, y)=0$.

Proposição 4.4. (GEORGESCU, 2007) Seja * uma t-norma e considere $Q$ uma relação fuzzy em $X$ fortemente completa. Então, se $Q$ é transitiva, $Q$ é quasi-transitiva.

Demonstração. Sejam $x, y, z \in X$ quaisquer.

$$
Q(z, y) * Q(y, x) \leq Q(z, x)
$$

por transitividade de $Q$. Logo,

$$
Q(z, y) * Q(y, x) * \neg Q(z, x) \leq Q(z, x) * \neg Q(z, x)=0 .
$$

Assim sendo, pela Proposição 4.3 .

$$
Q(z, y) * \neg Q(z, x) \leq \neg Q(y, x)
$$


Por outro lado, como $Q$ é fortemente completa,

$$
Q(x, z) * \neg Q(y, z) \leq Q(x, z) * Q(z, y) \leq Q(x, y),
$$

sendo o último passo por transitividade de $Q$. Portanto,

$$
\begin{aligned}
P_{Q}(x, z) * P_{Q}(z, y) & \leq Q(x, z) * \neg Q(z, x) * Q(z, y) * \neg Q(y, z)= \\
& =[Q(z, y) * \neg Q(z, x)] *[Q(x, z) * \neg Q(y, z)] \leq \\
& \leq \neg Q(y, x) * Q(x, y)= \\
& =P_{Q}(x, y) .
\end{aligned}
$$

A definição a seguir é análoga ao caso clássico.

Definição 4.7. Dada uma relação fuzzy $Q$, o seu fecho $*$-transitivo $Q^{*}$ é a relação fuzzy que possui as seguintes propriedades:

1. $Q^{*}$ é transitiva

2. $Q \subseteq Q^{*}$

3. se uma relação fuzzy $U$ é transitiva e $Q \subseteq U$, então $Q^{*} \subseteq U$

A unicidade segue da definição. Já a existência de um fecho transitivo para qualquer tnorma e qualquer relação fuzzy foi confirmada em Baets e Meyer (2003). A demonstração de tal fato foge do escopo deste trabalho.

\subsection{Definições Preliminares da Teoria de Escolha Fuzzy}

Os conceitos que foram introduzidos no Capítulo 3 podem ser estendidos para abarcar a possibilidade de proposições com valores de verdade entre [0,1] - sugerindo uma expansão dos conceitos de preferência revelada e função de escolha para preferência revelada fuzzy e função de escolha fuzzy.

É natural pensar que relações de preferência são, em muitas situações reais, vagas. Em Orlovsky (1977), é construída uma função de escolha a partir de uma relação de preferência fuzzy. No caso, os conjuntos das alternativas continuam sendo crisp, ou seja, $\mathcal{B}$ é uma família de conjuntos $S$ cujo o grau de pertinência de um elemento $x$ do conjunto universo a $S$ é 0 ou 1 . Um exemplo dado é o caso em há um conjunto de alternativas $S$, mas o valor de verdade da afirmação "a alternativa $x$ é tão boa quanto a alternativa $y$ ", para $x, y \in S$ não é estritamente 0 ou 1 , e especialistas são chamados para dar um peso entre 0 e 1 à afirmação. A partir dos pesos dados, mediante certas condições, a função de escolha de Orslovsky garante um conjunto crisp não-vazio de alternativas que são, no sentido dado pelo autor, não-dominadas. 
Vale lembrar, principalmente no contexto econômico, que essa imprecisão não é a mesma coisa que indiferença. Isso pode ser visto da seguinte maneira: considere um consumidor que é indiferente entre, por exemplo, uma maçã e uma banana. Um mecanismo aleatório irá determinar qual das frutas o consumidor receberá com uma probabilidade, que pode ser ajustada, $\alpha$ de ser a maçã e a probabilidade $1-\alpha$ de ser a banana. Como o consumidor é indiferente, ele não se importaria com o valor de $\alpha$, ou seja, não perderia tempo ajustando tal mecanismo. Essa insensitividade a $\alpha$ não ocorreria necessariamente no caso de uma preferência vaga. Se esse consumidor prefere maçã a banana com um grau de 0.7 e banana a maçã com um grau de 0.5 , provavelmente iria querer ajustar o mecanismo se $\alpha$ tivesse um valor de 0.1 . Se é possível determinar com precisão um valor para $\alpha$ baseado nas preferências fuzzy de tal maneira que o consumidor ficasse completamente satisfeito com esse valor ou como se daria uma relação entre a satisfação do consumidor e o valor de $\alpha$ é matéria para investigação que não cabe neste trabalho.

Por sua vez, o trabalho de Banerjee (1995) permite que tanto as preferências quanto a escolha sejam vagas. Porém, o espaço de escolha, ou seja, se uma alternativa está ou não disponível para ser escolhida, continua sendo crisp. A justificativa do autor é que, dada uma opção $x$ num conjunto $S$ de alternativas disponíveis, um tomador de decisões pode ter uma inclinação de 0.8 a escolher essa alternativa. Além disso, como sugere o autor, isso pode ser, pelo menos potencialmente, observado - como, por exemplo, pelo grau de entusiasmo que o tomador de decisão demonstre. Partindo da sugestão do autor, a inclinação à escolha de um elemento $t \in\{x, y, z\}$ poderia ser medido mostrando a um entrevistado as alternativas $x, y$ e $z$ que ele poderia receber e, após sortear uma delas, perguntar qual seu grau de satisfação entre 0 e 1 pela alternativa ter sido a sorteada.

Pensando num contexto econômico mais tangível, é possível imaginar a situação de um consumidor com uma quantidade $r$ de dinheiro em um estádio de futebol onde o preço da cerveja é $0<p \leq r$ e ele deve tomar a decisão de comprar ou não. Nesse exemplo, o espaço de escolha $(X, \mathcal{B})$ é formado por $X=\{0,1\} \times[0, r]$, que represente a compra ou não da cerveja e o dinheiro que irá sobrar. Para cada preço, há uma restrição diferente, ou seja, cada conjunto $S_{p} \in \mathcal{B}$ tem como elementos as duas opções disponíveis (de comprar ou não) dependendo do preço. Assim sendo, $S_{p}=\{(0, r),(1, r-p)\}$. Se esse consumidor for representado por uma função de escolha clássica, a não ser que ele sempre compre cerveja ou nunca compre, independentemente de $p$, surge uma situação potencialmente absurda. Caso $p^{\prime}$ seja o preço máximo que ele está disposto a pagar pela cerveja, ele já não a compraria por $p^{\prime}+0.01^{11}$. Essa aparente contradição se dá mesmo pensando em uma situação de indiferença, caso ele não fosse sempre indiferente entre comprar ou não. No entanto, se é permitida uma escolha fuzzy, esse "paradoxo" é resolvido, uma vez que a inclinação à escolha de se comprar a cerveja pode mudar

11 O preço máximo que um consumidor está disposto a pagar por um bem é denominado, em economia, prȩ̧o de reserva. 
suavemente. O argumento de que a escolha terá que ser feita de uma maneira ou de outra não invalida a "fuzzyficação" da mesma, já que ela trata de uma tendência a efetuar a compra. Isso significa que a escolha observada de fato somente diz que a propensão a fazê-la era diferente de zero. Além disso, é de se notar que essa inclinação à compra é diferente, tanto no tratamento matemático quanto concretamente, de uma aleatorização baseada em preferências da escolha. A Figura 1 ilustra ambas as situações de tendência à compra.

Figura 1 - "Fuzzyficação" da inclinação à compra
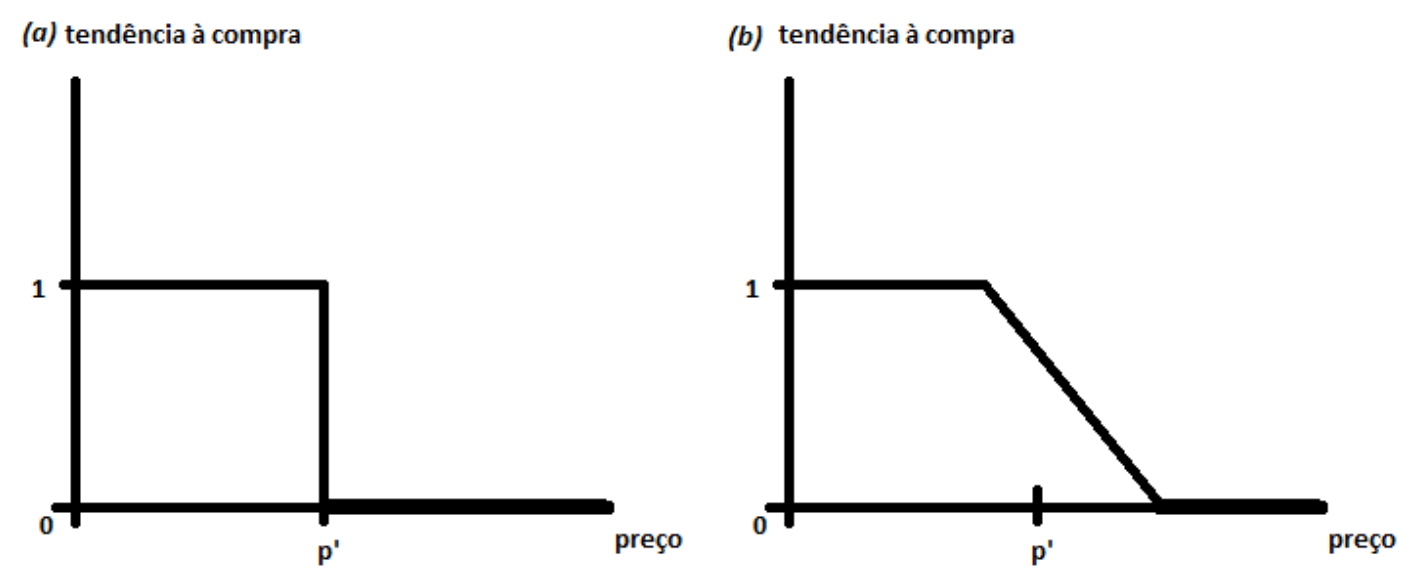

(a): caso onde a tendência é 1 ou 0 e (b): caso onde a tendência pode ter valores entre 0 e 1. Fonte: o autor

Por fim, em Georgescu (2007), além da escolha e preferências, é permitido vagueza também ao espaço das alternativas. Ou seja, $\mathcal{B}$ passa a ser uma família de conjuntos fuzzy não-zeros de $X$. Segundo a autora, o grau de pertencimento de um elemento a um conjunto de $\mathcal{B}$ poderia representar atributos ou critérios vagos. Assim sendo, para $S \in \mathcal{B}$, $S(x)$ seria o grau de disponibilidade de $x$. O exemplo dado pela autora é de $X$ como um conjunto de elementos onde a cor verde aparece em quatro nuances $a, b, c$ e $d$; e o atributo "verde" é um conjunto fuzzy de $X, V$. Cada nuance é um grau de intensidade da cor verde entre 0 e 1 . Respectivamente, $0, \frac{1}{3}, \frac{2}{3}$ e 1 . Logo, $V(x)=\frac{1}{3}$ indica que o objeto $x$ possui a nuance $b$. No entanto, em qual situação econômica concreta a "fuzzyficação" do espaço de escolha se aplicaria?

É possível pensar no seguinte problema: um agente econômico deve elaborar um orçamento, porém seu gasto deve ser determinado antes que sua renda se concretize. $\mathrm{Ou}$ seja, há uma vagueza sobre a renda disponível. Por exemplo, seja $X=R_{+}^{2}$ o conjunto de cestas formadas por dois bens. Assim, $(x, y) \in X$ representa uma cesta. No entanto, o nível de renda ainda não está determinado com certeza. Desse modo, cada $S \in \mathcal{B}$ representa uma restrição orçamentária vaga diferente. Nesse caso, $S(x, y)$ é o valor verdade entre 0 e 1 da afirmação "a cesta $(x, y)$ está disponível para compra”. A Figura 2 representa 
graficamente um dado $S \in \mathcal{B}$.

Figura 2 - Vagueza na renda

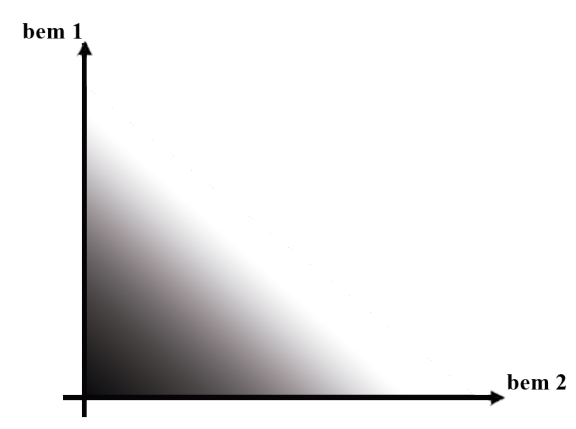

A disponibilidade da cesta de bens vai de 1 (cor preta) até 0 (cor branca). Fonte: o autor

Pensando sobre uma decisão concreta, por exemplo, sobre um orçamento no caso de um governo, parece ser mais promissor vê-la pela ótica de conjuntos fuzzy ao invés de baseada em probabilidades de arrecadação e valor esperado do retorno dos gastos (seja qual for a medida de retorno). Isso porque se trata de um processo em que decisãoconsequência ocorre apenas uma vez. Tal escolha se difere, por exemplo, da determinação da melhor jogada perante uma situação num jogo de poker, uma vez que, dada a natureza do jogo, essa situação se repete.

Este trabalho segue a linha de Georgescu (2007), tanto na questão da vagueza da disponibilidade dos elementos do conjunto $X$ quanto nas definições que serão vistas a seguir.

Definição 4.8. Dados um conjunto universo $X$ e $\mathcal{B}$ uma subfamília não-vazia de $\mathcal{F}(X)$, o par $(X, \mathcal{B})$ é denominado espaço de escolha fuzzy.

Definição 4.9. Dados um espaço de escolha fuzzy $(X, \mathcal{B})$, uma função de escolha fuzzy é um mapa $C: \mathcal{B} \rightarrow \mathcal{F}(X)$ tal que, para cada $S \in \mathcal{B}, C(S)$ é não-zero e $C(S) \subseteq S$.

As relações definidas a seguir são análogas às relações da teoria clássica.

Definição 4.10. Dados um espaço de escolha fuzzy $(X, \mathcal{B})$, uma função de escolha fuzzy $C$ e uma t-norma $*$, podem ser definidas as seguintes relações fuzzy em $X$ :

- $R(x, y)=\bigvee_{S \in \mathcal{B}}[C(S)(x) * S(y)]$ 
- $P(x, y)=R(x, y) * \neg R(y, x)$;

- $\widetilde{P}(x, y)=\bigvee_{S \in \mathcal{B}}[C(S)(x) * S(y) * \neg C(S)(y)]$

- $W$ é o fecho transitivo de $R$;

- $P^{*}$ é o fecho transitivo de $\widetilde{P}$.

Se $\mathcal{B}$ contém todos os conjuntos $[x, y], x, y \in X$, pode-se definir também:

- $\bar{R}(x, y)=C([x, y])(x)$

Da mesma maneira, seguem as definições:

Definição 4.11. Dados uma t-norma $*$, um espaço de escolha fuzzy $(X, \mathcal{B})$ e uma função de escolha fuzzy $C$, a imagem de $C$ é o mapa $\widehat{C}: \mathcal{B} \rightarrow \mathcal{F}(X)$ definido por $\widehat{C}(S)(x)=$ $S(x) * \bigwedge_{y \in X}[S(y) \longrightarrow R(x, y)]$.

Definição 4.12. Dados um espaço de escolha fuzzy $(X, \mathcal{B})$ e uma função de escolha fuzzy $C, C$ é denominada normal quando $C=\widehat{C}$. Isto é, para todo $S \in \mathcal{B}$ e todo $x \in X$, $C(S)(x)=\widehat{C}(S)(x){ }^{12}$

Com essas ferramentas, é possível, então, elaborar a formalização no contexto fuzzy dos axiomas apresentados no Capítulo 2, sendo essa formalização análoga à feita na Seção 3.2 .

Dados um espaço de escolha fuzzy, $(X, \mathcal{B})$, uma função de escolha fuzzy $C$, uma tnorma $*$ e as relações apresentadas em 4.10 , $C$ pode cumprir os axiomas e condições de racionalidade a seguir.

- WAFRP (Axioma Fraco de Preferência Fuzzy Revelada):

$$
\widetilde{P}(x, y) \leq \neg R(y, x) ; \forall x, y \in X
$$

- SAFRP (Axioma Forte de Preferência Fuzzy Revelada:

$$
P^{*}(x, y) \leq \neg R(y, x) ; \forall x, y \in X
$$

- WFCA (Axioma Fuzzy de Congruência Fraco):

$$
S(x) * C(S)(y) * R(x, y) \leq C(S)(x) ; \forall S \in \mathcal{B}, \forall x, y \in X
$$

- SFCA (Axioma Fuzzy de Congruência Forte):

$$
S(x) * C(S)(y) * W(x, y) \leq C(S)(x) ; \forall S \in \mathcal{B}, \forall x, y \in X
$$

\footnotetext{
12 A questão de normalidade no caso fuzzy, assim como no caso clássico, é tratada no anexo $\mathrm{A}$
} 
- Condição $F a$ 13.

$$
I(S, T) * S(x) * C(T)(x) \leq C(S)(x) ; \forall S, T \in \mathcal{B}, \forall x \in X
$$

- Condição $F \beta$

$$
I(S, T) * C(S)(x) * C(S)(y) \leq \rho(C(T)(x), C(T)(y)) ; \forall S, T \in \mathcal{B}, \forall x, y \in X
$$

13 É utilizado o grau de inclusão de um conjunto fuzzy em outro, definido, para uma dada t-norma, como:

$$
I(S, T)=\bigwedge_{x \in X}\left[S(x) \rightarrow_{*} T(x)\right]
$$




\section{Resultados}

Este capítulo busca estabelecer, no contexto da teoria de conjuntos fuzzy, resultados semelhantes àqueles alcançados no teorema de Arrow-Sen. Para isso, seguindo a linha de Georgescu (2007) e Martinetti et al. (2014), trabalhos nos quais este Capítulo é baseado, são elaborados as hipóteses a seguir acerca de um espaço de escolha fuzzy $(X, \mathcal{B})$.

H1: para todo $S \in \mathcal{B}$, existe $x \in X$ tal que $C(S)(x)=S(x)=1$.

WH1: para todo $S \in \mathcal{B}$, existe $x \in X$ tal que $C(S)(x)=S(x)>0$.

H2: os conjuntos $[x],[x, y]$ e $[x, y, z]$ estão inclusos em $\mathcal{B}$ para quaisquer $x, y, z \in X$. Vale notar que H2 é semelhante à hipótese $\mathrm{H}$ da teoria da escolha clássica. A hipótese H1, elaborada por Georgescu (2007), é automaticamente garantida no caso clássico. Por sua vez, a WH1, versão mais fraca de H1 que foi elaborada por Martinetti et al. (2014), também é automaticamente garantida no caso clássico.

Para todas as demonstrações deste capítulo, assume-se uma dada t-norma contínua pela esquerda. A não ser que esteja explicitado, essa é a única exigência sobre ela.

Para começar, serão investigadas as conexões entre WFCA, SFCA, WAFRP e SAFRP.

Proposição 5.1. Sejam um espaço de escolha fuzzy $(X, \mathcal{B})$ e uma função de escolha fuzzy C. Então,

(i) Se C cumpre SAFRP, C cumpre WAFRP;

(ii) Se C cumpre SFCA, C cumpre WFCA.

Demonstração. Ambas afirmações são imediatas, uma vez que:

(i) $\widetilde{P} \subseteq P^{*}$;

(ii) $R \subseteq W$.

Lema 5.1. (GEORGESCU, 2007) Sejam um espaço de escolha fuzzy $(X, \mathcal{B})$ que cumpre WH1 e H2 e uma função de escolha fuzzy $C$. Então, se $C$ cumpre WFCA, $R$ é transitiva.

Demonstração. Para quaisquer $x, y, z \in X$, considere o conjunto $T=[x, y, z]$. É preciso provar que $R(x, z) * R(z, y) \leq R(x, y)$. Por WH1, há três casos:

(i) $C(T)(x)=1$.

$$
R(x, y)=\bigvee_{S \in \mathcal{B}}[C(S)(x) * S(y)] \geq C(T)(x) * T(y)=1
$$


(ii) $C(T)(y)=1$. Usando WFCA,

$$
R(z, y)=R(z, y) * C(T)(y) * T(z) \leq C(T)(z)
$$

Logo, usando novamente WFCA,

$$
R(x, z) * R(z, y) \leq R(x, z) * C(T)(z) * T(x) \leq C(T)(x)=C(T)(x) * T(y) \leq R(x, y)
$$

(iii) $C(T)(z)=1$. Usando WFCA,

$$
\begin{aligned}
R(x, z) * R(z, y) & \leq R(x, z)=R(x, z) * C(T)(z) * T(x) \leq \\
& \leq C(T)(x)=C(T)(x) * T(y) \leq \\
& \leq R(x, y) .
\end{aligned}
$$

Proposição 5.2. (GEORGESCU, 2007) Sejam $(X, \mathcal{B})$ um espaço de escolha fuzzy que cumpre WH1 e H2 e C uma função de escolha fuzzy. Então, se C cumpre WFCA, C cumpre SFCA.

Demonstração. Pelo lema 5.1 e pela definição 4.7, $R=W$. Logo, se $C$ cumpre WFCA, para qualquer $S \in \mathcal{B}$ e quaisquer $x, y \in X$,

$$
C(S)(y) * S(x) * W(x, y)=C(S)(y) * S(x) * R(x, y) \leq C(S)(x) .
$$

Proposição 5.3. (MARTINETTI et al., 2014) Sejam $(X, \mathcal{B})$ um espaço de escolha fuzzy que cumpre WH1 e H2 e C uma função de escolha fuzzy. Então, se C cumpre WAFRP, C cumpre SAFRP.

Demonstração. É suficiente demonstrar que $\widetilde{P}$ é transitiva, pois, assim sendo, pela definição 4.7, se $C$ cumpre WAFRP, para quaisquer $x, y \in X$,

$$
P^{*}(x, y)=\widetilde{P}(x, y) \leq \neg R(y, x) .
$$

Para quaisquer $x, y, z \in X$, considere o conjunto $T=[x, y, z]$. É preciso mostrar que $\widetilde{P}(x, z) * \widetilde{P}(z, y) \leq \widetilde{P}(x, y)$. Considerando WAFRP,

$$
\begin{aligned}
\widetilde{P}(x, z) & \leq \neg R(z, x) \leq \\
& \leq \neg \bigvee_{S \in \mathcal{B}}[C(S)(z) * S(x)]= \\
& =\bigwedge_{S \in \mathcal{B}} \neg[C(S)(z) * S(x)] \leq \\
& \leq \neg[C(T)(z) * T(x)]=\neg C(T)(z) .
\end{aligned}
$$


De maneira análoga,

$$
\widetilde{P}(z, y) \leq \neg C(T)(y) .
$$

Por outro lado,

$$
\begin{aligned}
\widetilde{P}(x, y) & =\bigvee_{S \in \mathcal{B}}[C(S)(x) * S(y) * \neg C(S)(y)] \geq \\
& \geq C(T)(x) * T(y) * \neg C(T)(y)=C(T)(x) * \neg C(T)(y) .
\end{aligned}
$$

Por WH1, há três casos a serem analisados.

(i) $C(T)(x)=1$.

$$
\begin{aligned}
\widetilde{P}(x, z) * \widetilde{P}(z, y) & \leq \neg C(T)(z) * \neg C(T)(y) \leq & & \text { por 5.1) e 5.2 } \\
& \leq C(T)(x) * \neg C(T)(y) \leq & & \\
& \leq \widetilde{P}(x, y) ; & & \text { por 5.3 }
\end{aligned}
$$

(ii) $C(T)(y)=1$. Usando 5.2$)$ :

$$
\widetilde{P}(x, z) * \widetilde{P}(z, y) \leq \widetilde{P}(z, y) \leq \neg C(T)(y)=0 ;
$$

(iii) $C(T)(z)=1$. Usando 5.1$)$ :

$$
\widetilde{P}(x, z) * \widetilde{P}(z, y) \leq \widetilde{P}(x, z) \leq \neg C(T)(z)=0 .
$$

Proposição 5.4. (MARTINETTI et al., 2014) Sejam um espaço de escolha fuzzy $(X, \mathcal{B}) e$ $C$ uma função de escolha fuzzy. Então, se C cumpre WFCA, C também cumpre WAFRP. Demonstração. Suponha que $C$ não cumpra WAFRP. Assim sendo, existem $x, y \in X$ tais que $\widetilde{P}(x, y)>\neg R(y, x)$. Logo, pela Proposição 4.3 .

$$
\begin{aligned}
0 & <\widetilde{P}(x, y) * R(y, x)= \\
& =\bigvee_{S \in \mathcal{B}}[C(S)(x) * S(y) * \neg C(S)(y)] * R(y, x)= \\
& =\bigvee_{S \in \mathcal{B}}[C(S)(x) * S(y) * \neg C(S)(y) * R(y, x)]
\end{aligned}
$$

Portanto, existe um $S \in \mathcal{B}$ tal que:

$$
C(S)(x) * S(y) * \neg C(S)(y) * R(y, x)>0 .
$$

Usando novamente a Proposição 4.3 .

$$
C(S)(x) * S(y) * R(y, x)>\neg \neg C(S)(y) \geq C(S)(y) .
$$

Ou seja, $C$ não cumpre WFCA. 
Abaixo segue uma demonstração alternativa mais direta:

Demonstração. Sejam $x, y \in X$ quaisquer. Considere um $S \in \mathcal{B}$ qualquer. Usando WFCA e a Proposição 4.3 .

$$
C(S)(x) * S(y) * \neg C(S)(y) * R(y, x) \leq C(S)(y) * \neg C(S)(y)=0
$$

Como $S$ é arbitrário,

$$
\begin{aligned}
0 & =\bigvee_{S \in \mathcal{B}}[C(S)(x) * S(y) * \neg C(S)(y) * R(y, x)]= \\
& =\bigvee_{S \in \mathcal{B}}[C(S)(x) * S(y) * \neg C(S)(y)] * R(y, x)= \\
& =\widetilde{P}(x, y) * R(y, x) .
\end{aligned}
$$

Logo, usando novamente a Proposição 4.3 .

$$
\widetilde{P}(x, y) \leq \neg R(y, x) .
$$

Proposição 5.5. (MARTINETTI et al., 2014) Sejam um espaço de escolha fuzzy $(X, \mathcal{B})$ e uma função de escolha fuzzy $C$. Considere que a t-norma * induz uma negação forte, ou seja, $\neg \neg a=a$. Então, se C cumpre WAFRP, C cumpre WFCA.

Demonstração. Suponha que $C$ não cumpra WFCA. Então, existe $\bar{S} \in \mathcal{B}$ e $x, y \in X$ tais que:

$$
C(\bar{S})(x) * \bar{S}(y) * R(y, x)>C(\bar{S})(y)=\neg \neg C(\bar{S})(y)
$$

pelo fato da negação ser forte. Usando a Proposição 4.3 na primeira linha,

$$
\begin{aligned}
0 & <C(\bar{S})(x) * \bar{S}(y) * \neg C(\bar{S})(y) * R(y, x) \leq \\
& \leq \bigvee_{S \in \mathcal{B}}[C(S)(x) * S(y) * \neg C(S)(y)] * R(y, x)= \\
& =\widetilde{P}(x, y) * R(y, x) .
\end{aligned}
$$

Logo, novamente pela pela Proposição 4.3 .

$$
\widetilde{P}(x, y)>\neg R(y, x) .
$$

Portanto, $C$ não cumpre WAFRP.

Novamente, a proposição pode ser demonstrada de maneira direta.

Demonstração. Sejam $x, y \in X$ e $S \in \mathcal{B}$ quaisquer. Por WAFRP e Proposição 4.3.

$$
\widetilde{P}(y, x) * R(x, y)=0 \text {. }
$$


Logo, usando a definição de $\widetilde{P}$,

$$
C(S)(y) * S(x) * R(x, y) * \neg C(S)(x) \leq \widetilde{P}(y, x) * R(x, y)=0
$$

Portanto, usando novamente 4.3 e o fato de * gerar uma negação forte,

$$
C(S)(y) * S(x) * R(x, y) \leq \neg \neg C(S)(x)=C(S)(x) .
$$

O exemplo a seguir mostra que, mesmo que as hipóteses H1 e H2 sejam cumpridas, não basta a t-norma ser contínua à esquerda para que WAFRP implique WFCA.

Exemplo 5.1. Considere o espaço de escolha fuzzy $X=\{x, y\}$ e a família de conjuntos fuzzy $\mathcal{B}=\{[x],[y],[x, y], S\}$, sendo que $S(x)=0.7$ e $S(y)=1$. Além disso, considere a função de escolha fuzzy $C$ tal que $C([x])(x)=C([y])(y)=1, C([x, y](x)=0.7$, $C([x, y])(y)=1, C(S)(x)=0.5$ e $C(S)(y)=1$. Note que esse espaço de escolha fuzzy cumpre H1 e H2. Considere * a t-norma de Gödel. Calculando a relação $R$, obtém-se: $R(x, y)=0.7$ e $R(y, x)=1$. Por outro lado,

$$
\begin{aligned}
& \widetilde{P}(x, y)=\bigvee_{T \in \mathcal{B}}[C(T)(x) * T(y) * \neg C(T)(y)]=0 \\
& \widetilde{P}(y, x)=\bigvee_{T \in \mathcal{B}}[C(T)(y) * T(x) * \neg C(T)(x)]=0,
\end{aligned}
$$

uma vez que, como * é a t-norma de Gödel, $\neg a=0$ quando a $>0$. Assim sendo, $C$ cumpre WAFRP. No entanto,

$$
C(S)(y) * S(x) * R(x, y)=1 * 0.7 * 0.7=0.7>0.5=C(S)(x)
$$

Ou seja, C não cumpre WFCA.

Lema 5.2. (GEORGESCU, 2007) Sejam um espaço de escolha fuzzy $(X, \mathcal{B})$ que cumpre as hipóteses WH1 e H2 e uma função de escolha fuzzy C. Então:

(i) $\bar{R} \subseteq R$;

(ii) $R$ e $\bar{R}$ são fortemente reflexivas e fortemente totais.

Demonstração. (i) Para quaisquer $x, y \in X$,

$$
\bar{R}(x, y)=C([x, y])(x) *[x, y](y) \leq \bigvee_{S \in \mathcal{B}}[C(S)(x) * S(y)]=R(x, y)
$$

(ii) Para quaisquer $x, y \in X$,

$$
R(x, x) \geq \bar{R}(x, x)=C([x])(x)=1,
$$


por WH1. Por outro lado, se $C([x, y])<1$, também por WH1, $C([y, x])=1$. Logo,

$$
R(y, x) \geq \bar{R}(y, x)=C([y, x])=1
$$

. Caso $C([x, y])=1$

$$
R(x, y) \geq \bar{R}(x, y)=C([x, y])=1
$$

A proposição a seguir é uma demonstração alternativa que leva em conta as propriedades do fecho transitivo.

Proposição 5.6. Sejam um espaço de escolha fuzzy $(X, \mathcal{B})$ que cumpre WH1 e H2 e uma função de escolha fuzzy $C$. Então, se C cumpre WFCA, C cumpre SAFRP.

Demonstração. Como $C$ cumpre WFCA, as seguinte afirmações são verdadeiras:

(a) $P$ é transitiva.

Pelos Lemas 5.1 e 5.2 e a Proposição 4.4.

(b) $\widetilde{P} \subseteq P$

Pelo Lema 5.2, para quaisquer $x, y \in X$, existem duas possibilidades: $R(x, y)=1$ ou $R(y, x)=1$. Suponha $R(x, y)=1$. Nesse caso, para qualquer $S \in \mathcal{B}$,

$C(S)(x) * S(y) * \neg C(S)(y) * R(y, x) \leq C(S)(x) * S(y) * R(y, x) * \neg[C(S)(x) * S(y) * R(y, x)]=0$,

levando em conta o WFCA e a proposição 4.3. Logo, também pela Proposição 4.3 . para todo $S \in \mathcal{B}$,

$$
C(S)(x) * S(y) * \neg C(S)(y) \leq \neg R(y, x)=R(x, y) * \neg R(y, x)=P(x, y) .
$$

Como $S$ é arbitrário, $\widetilde{P}(x, y) \leq P(x, y)$. Suponha agora que $R(y, x)=1$. Assim sendo, para todo $S \in \mathcal{B}$,

$$
C(S)(x) * S(y) * \neg C(S)(y) \leq C(S)(x) * S(y) * \neg[C(S)(x) * S(y) * R(y, x)]=0,
$$

usando a WFCA e a Proposição 4.3 . Logo, $\widetilde{P}(x, y)=0$.

Suponha que $C$ não cumpra o SAFRP. Nesse caso, existem $\tilde{x}, \tilde{y} \in X$ tais que $P^{*}(\tilde{x}, \tilde{y})>$ $\neg R(\tilde{y}, \tilde{x})$. Assim sendo,

$$
P(\tilde{x}, \tilde{y})=R(\tilde{x}, \tilde{y}) * \neg R(\tilde{y}, \tilde{x}) \leq \neg R(\tilde{y}, \tilde{x})<P^{*}(\tilde{x}, \tilde{y})
$$

Uma contradição, levando em conta as afirmações (a), (b) e o fato de que $P^{*}$ é o fecho transitivo de $\widetilde{P}$.

A Figura 3 sintetiza as proposições provadas acima.

A seguir será investigada a conexão entre o WFCA, a normalidade de $C$ e a regularidade de $R$. 


\section{Figura 3 - WFCA, SFCA, WAFRP e SAFRP}

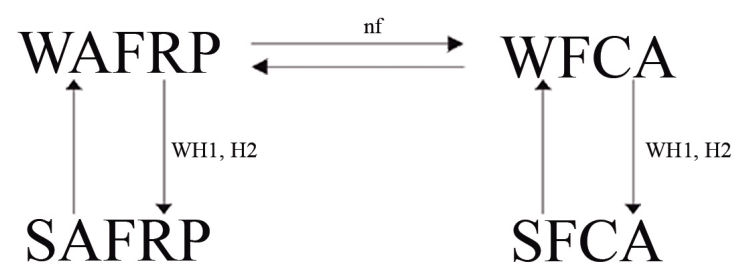

nf: t-norma gera negação forte

Fonte: o autor.

Proposição 5.7. (GEORGESCU, 2007)Sejam $(X, \mathcal{B})$ um espaço de escolha fuzzy que cumpre WH1 e H2 e C uma função de escolha fuzzy. Então, se C cumpre WFCA, $R$ é regular.

Demonstração. Pelos Lemas 5.1 e 5.2 .

Lema 5.3. Sejam $(X, \mathcal{B})$ um espaço de escolha fuzzy que cumpre $H 1$ e $C$ uma função de escolha fuzzy. Então, se $C$ cumpre $W F C A, \widehat{C} \subseteq C$.

Demonstração. Seja $x \in X$ e $S \in \mathcal{B}$. Por H1, existe $z \in S$ tal que $C(S)(z)=S(z)=1$. Logo,

$$
\begin{aligned}
\widehat{C}(S)(x) & =S(x) * \bigwedge_{y \in X}[S(y) \rightarrow R(x, y)] \leq \\
& \leq S(x) *[S(z) \rightarrow R(x, z)]= \\
& =S(x) * R(x, z) * C(S)(z) \leq \\
& \leq C(S)(x) .
\end{aligned}
$$$$
\text { por WFCA }
$$

O exemplo a seguir mostra que a hipótese $\mathrm{H} 1$ não pode ser substituída pela hipótese WH1 para que o Lema 5.3 seja válido. 
Exemplo 5.2. Considere o espaço de escolha fuzzy $X=\{x, y\}$ e a família de conjuntos fuzzy $\mathcal{B}=\{[x],[y],[x, y], S\}$, sendo que $S(x)=0.9$ e $S(y)=1$. Além disso, considere a função de escolha fuzzy $C$ tal que $C([x])(x)=C([y])(y)=1, C([x, y](x)=1$, $C([x, y])(y)=0.5, C(S)(x)=0.8$ e $C(S)(y)=0.5$. Note que esse espaço de escolha fuzzy cumpre WH1 e H2. Considere * a t-norma de Gödel. Calculando a relação $R$, obtém-se: $R(x, y)=1$ e $R(y, x)=0.5$. Para os conjuntos $[x]$ e $[y]$, é trivial que vale a desigualdade da WFCA. Já para os outros dois, tem-se que:

$$
\begin{aligned}
& C([x, y])(x) *[x, y](y) * R(y, x)=1 * 1 * 0.5 \leq C([x, y])(y) \\
& C([x, y])(y) *[x, y](x) * R(x, y)=0.5 * 1 * 1 \leq C([x, y])(x) \\
& C(S)(x) * S(y) * R(y, x)=0.8 * 0.5 * 0.5 \leq 0.5=C(S)(y) \\
& C(S)(y) * S(x) * R(x, y)=0.5 * 0.9 * 1 \leq 0.8=C(S)(x) .
\end{aligned}
$$

Ou seja, C cumpre WFCA. No entanto,

$$
\widehat{C}(S)(x)=S(x) * \bigwedge_{z \in X}[S(z) \rightarrow R(x, z)]=0.9>0.8=C(S)(x) .
$$

Lema 5.4. (GEORGESCU, 2007)Sejam $(X, \mathcal{B})$ um espaço de escolha fuzzy e $C$ uma função de escolha fuzzy. Se * é a t-norma de Gödel, então $C \subseteq \widehat{C}$.

Demonstração. Seja $x \in X$ e $S \in \mathcal{B} . C(S)(x) \leq S(x)$ por definição. Além disso, para qualquer $y \in X, C(S)(x) * S(y) \leq R(x, y)$. Logo, $C(S)(x) \leq S(y) \rightarrow R(x, y)$ e, como $y$ é arbitrário, $C(S)(x) \leq \bigwedge_{y \in X}[S(y) \rightarrow R(x, y)]$. Portanto, para a t-norma de Gödel,

$$
C(S)(x)=C(S)(x) * C(S)(x) \leq S(x) * \bigwedge_{y \in X}[S(y) \rightarrow R(x, y)]=\widehat{C}(S)(x) .
$$

O exemplo abaixo mostra que, mesmo o espaço de escolha fuzzy cumprindo H1 e H2 e a função de escolha cumprindo WFCA, se a t-norma não for a de Gödel, o Lema 5.4 pode não valer.

Exemplo 5.3. Considere o espaço de escolha fuzzy $X=\{x, y\}$ e a família de conjuntos fuzzy $\mathcal{B}=\{[x],[y],[x, y], S\}$, sendo que $S(x)=0.3$ e $S(y)=1$. Além disso, considere a função de escolha fuzzy $C$ tal que $C([x])(x)=C([y])(y)=1, C([x, y](x)=0.25$, $C([x, y])(y)=1, C(S)(x)=0.25$ e $C(S)(y)=1$. Note que esse espaço de escolha fuzzy cumpre H1 e H2. Considere * a t-norma produto. Calculando a relação $R$, obtém-se: $R(x, y)=0.25$ e $R(y, x)=1$. Para os conjuntos $[x] e[y]$, é trivial que vale a desigualdade da WFCA. Já para os outros dois, tem-se que:

$$
\begin{gathered}
C([x, y])(x) *[x, y](y) * R(y, x)=0.25 * 1 * 1 \leq 1=C([x, y])(y) \\
C([x, y])(y) *[x, y](x) * R(x, y)=1 * 1 * 0.25 \leq 0.25=C([x, y])(x)
\end{gathered}
$$




$$
\begin{gathered}
C(S)(x) * S(y) * R(y, x)=0.25 * 1 * 1 \leq 1=C(S)(y) \\
C(S)(y) * S(x) * R(x, y)=1 * 0.3 * 0.25 \leq 0.25=C(S)(x) .
\end{gathered}
$$

Ou seja, C cumpre WFCA. Mesmo assim,

$$
C(S)(x)=0.25>0.3 * 0.25=S(x) * \bigwedge_{z \in X}[S(z) \rightarrow R(x, z)]=\widehat{C}(S)(x) .
$$

Proposição 5.8. Considere * a t-norma de Gödel. Sejam um espaço de escolha fuzzy $(X, \mathcal{B})$ que cumpre $H 1$ e uma função de escolha fuzzy $C$. Então, se C cumpre WFCA, C é normal.

Demonstração. Pelos Lemas 5.3 e 5.4 .

Proposição 5.9. (GEORGESCU, 2007) Sejam um espaço de escolha fuzzy $(X, \mathcal{B})$ e uma função de escolha fuzzy $C$. Então, se $C$ é normal e $R$ regular, $C$ cumpre WFCA.

Demonstração. Sejam $x, y \in X$ e $S \in \mathcal{B}$. Considere $z \in X$ qualquer. Pela transitividade de $R$,

$$
R(x, y) * C(S)(y) * S(z) \leq R(x, y) * R(y, z) \leq R(x, z)
$$

Logo, $R(x, y) * C(S)(y) \leq S(z) \rightarrow R(x, z)$ e, como $z$ é arbitrário,

$$
R(x, y) * C(S)(y) \leq \bigwedge_{z \in X}[S(z) \rightarrow R(x, z)]
$$

Assim sendo,

$$
\begin{aligned}
S(x) * C(S)(y) * R(x, y) & \leq S(x) * \bigwedge_{z \in X}[S(z) \rightarrow R(x, z)]= & & \text { por } 5.4 \\
& =\widehat{C}(S)(x)=C(S)(x) . & & \text { pela normalidade }
\end{aligned}
$$

A Figura 4 mostra os resultados obtidos.

Agora passa-se a investigar a conexão entre WFCA e as condições $F \alpha$ e $F \beta$. Mas, antes, são demonstrados dois lemas úteis.

Lema 5.5. Considere * a t-norma de Gödel. Sejam $(X, \mathcal{B})$ um espaço de escolha fuzzy que cumpre H2 e $C$ uma função de escolha fuzzy. Então, se $C$ cumpre $F \alpha, R=\bar{R}$.

Demonstração. Sejam $x, y \in X$. Considere $T=[x, y]$. Por um lado,

$$
\bar{R}(x, y)=C(T)(x) * T(y) \leq \bigvee_{S \in \mathcal{B}}[C(S)(x) * S(y)]=R(x, y)
$$


Figura 4 - WFCA, regularidade e normalidade

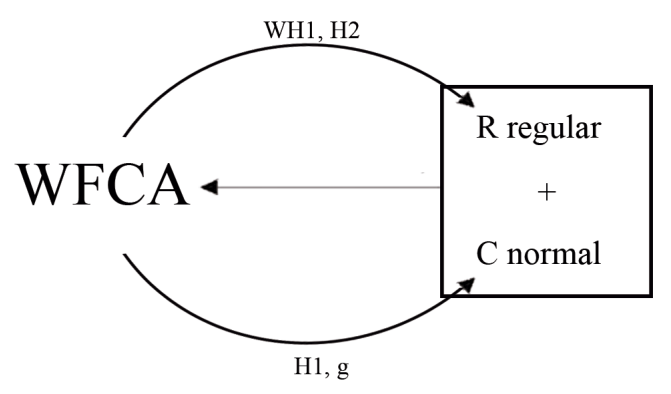

g: t-norma de Gödel

Fonte: o autor.

Por outro lado, considere um $S \in \mathcal{B}$ qualquer. Então, $I(T, S)=S(x) * S(y)$, uma vez que a t-norma é de Gödel. Portanto,

$$
\begin{aligned}
C(S)(x) * S(y) & =C(S)(x) * S(x) * S(y) \leq & & \text { pois } C(S)(x) \leq S(x) \\
& \leq C(S)(x) * I(T, S) * T(x) \leq & & \\
& \leq C(T)(x)=\bar{R}(x, y) & & \text { por } F \alpha
\end{aligned}
$$

Como $S$ é arbitrário,

$$
\bigvee_{S \in \mathcal{B}}[C(S)(x) * S(y)] \leq \bar{R}(x, y)
$$

Lema 5.6. Sejam $a, b, c \in[0,1]$ e * uma t-norma contínua pela esquerda. Então, se $a \leq b \rightarrow c, a * b \leq c$.

Demonstração. Usando a Proposição 4.3.

$$
a * b \leq(b \rightarrow c) * b \leq c .
$$

A proposição abaixo está em Martinetti et al. (2014). Porém, sem a demonstração. Há uma demonstração em Georgescu (2007), mas que utiliza a hipótese adicional H1.

Proposição 5.10. Considere * a t-norma de Gödel. Sejam $(X, \mathcal{B})$ um espaço de escolha fuzzy que cumpre H2 e $C$ uma função de escolha fuzzy. Então, se $C$ cumpre $F \alpha$ e $F \beta, C$ cumpre WFCA. 
Demonstração. Sejam $x, y \in X$ e $S \in \mathcal{B}$. Considere $T=[x, y]$. Como a t-norma é de Gödel, $I(T, S)=S(x) * S(y)$. Assim sendo,

$$
\begin{array}{rlrl}
C(S)(y) * S(x) * R(x, y) & =C(S)(y) * S(y) * S(x) * R(x, y)= & & \text { pois } C(S) \\
& =C(S)(y) * I(T, S) * R(x, y) \leq & & \\
& \leq T(y) * C(S)(y) * I(T, S) * I(T, S) * R(x, y) \leq & \\
& \leq C(T)(y) * R(x, y) * I(T, S)= & F \alpha \\
& =C(T)(y) * C(T)(x) * I(T, S) \leq & \text { Lema 5.5 } \\
& \leq C(S)(y) \rightarrow C(S)(x) . & F \beta
\end{array}
$$

Portanto, usando o Lema 5.6 .

$$
\begin{aligned}
C(S)(y) * S(x) * R(x, y) & =C(S)(y) * C(S)(y) * S(x) * R(x, y) \leq \\
& \leq C(S)(x) .
\end{aligned}
$$

O exemplo abaixo mostra que o fato da t-norma ser a de Gödel é essencial para a validade da Proposição 5.10 .

Exemplo 5.4. (MARTINETTI et al., 2014) Seja * uma t-norma qualquer que não seja a de Gödel. Considere o seguinte espaço de escolha fuzzy $X=\{x, y, z\}$ e $\mathcal{B}$ o conjunto de partes de $X$ excetuando o conjunto vazio. Além disso, considere a função escolha $C$ tal que $C([x])=C([y])=C([z])=1 ; C([x, y])(x)=1$ e $C([x, y])(y)=a ; C([x, z])(x)=a * b$ e $C([x, z])(z)=1 ; C([y, z])(y)=a$ e $C([y, z])(z)=1$; por fim, $C([x, y, z])(x)=a * b$, $C([x, y, z])(y)=a$ e $C([x, y, z])(z)=1$. Assuma $a, b \in] 0,1[$. Como a t-norma não é de Gödel, $a * b<a \wedge b \leq b$. Note que $(X, \mathcal{B})$ cumpre H1 e H2. Além disso, as condições $F \alpha$ e F $\beta$ estão satisfeitas (as contas são omitidas). Porém.

$$
R(x, y) *[x, y, z](x) * C([x, y, z])(y)=1 * 1 * a>a * b=C([x, y, z])(x) .
$$

Logo, C não cumpre WFCA.

Embora a proposição abaixo esteja em Martinetti et al. (2014), a demonstração a seguir parece ser mais intuitiva, além de ser direta.

Proposição 5.11. Sejam $(X, \mathcal{B})$ um espaço de escolha fuzzy que cumpre $H 1$ e $C$ uma função de escolha fuzzy. Então, se C cumpre WFCA, C cumpre F $\alpha$.

Demonstração. Sejam $x \in X$ e $S, T \in \mathcal{B}$. Por H1, existe $y \in S$ tal que $C(S)(y)=S(y)=$ 1. Assim sendo,

$$
\begin{array}{rlrl}
I(S, T) * C(T)(x) * S(x) & \leq[S(y) \rightarrow T(y)] * C(T)(x) * S(x)= & & \\
& =T(y) * C(T)(x) * S(x) * C(S)(y) \leq & \text { por } S(y)=C(S)(y)=1 \\
& \leq R(x, y) * S(x) * C(S)(y) \leq & \\
& \leq C(S)(x) & & \text { por WFCA }
\end{array}
$$


O exemplo a seguir mostra que H1 não pode ser substituído por WH1 para que a Proposição 5.11 seja válida.

Exemplo 5.5. Considere a seguinte espaço da escolha fuzzy: $X=\{x, y\}$ e a família de conjuntos fuzzy $\mathcal{B}=\{[x],[y],[x, y], S\}$, sendo que $S(x)=1$ e $S(y)=0.5$. Além disso, considere a função de escolha fuzzy $C$ tal que $C([x])(x)=1=C([y])(y)=1$, $C([x, y](x)=0.8, C([x, y])(y)=1, C(S)(x)=0.5$ e $C(S)(y)=0.5$. Note que esse espaço de escolha fuzzy cumpre WH1 e H2. Considere * a t-norma de Gödel. Calculando a relação $R$, obtém-se: $R(x, y)=0.8$ e $R(y, x)=1$. Para os conjuntos $[x]$ e $[y]$, é trivial que vale a desigualdade da WFCA. Já para os outros dois, tem-se que:

$$
\begin{gathered}
C([x, y])(x) *[x, y](y) * R(y, x)=0.8 * 1 * 1=0.8 \leq 1=C([x, y])(y) ; \\
C([x, y])(y) *[x, y](x) * R(x, y)=1 * 1 * 0.8=0.8=C([x, y])(x) \\
C(S)(x) * S(y) * R(y, x)=0.5 * 0.5 * 1=0.5=C(S)(y) \\
C(S)(y) * S(x) * R(x, y)=0.5 * 1 * 0.8=0.5=C(S)(x) .
\end{gathered}
$$

Ou seja, C cumpre WFCA. Mesmo assim,

$$
I(S, T) * C(T)(x) * S(x)=1 * 0.8 * 1=0.8>0.5=C(S)(x) .
$$

Portanto, C não cumpre F $\alpha$.

No trabalho de Martinetti et al. (2014), a hipótese H2 é utilizada para provar a proposição abaixo. A demonstração a seguir mostra que essa hipótese é desnecessária.

Proposição 5.12. Considere * a t-norma de Gödel. Sejam $(X, \mathcal{B})$ um espaço de escolha fuzzy e C uma função de escolha fuzzy. Então, se C cumpre WFCA, C cumpre F $\beta$.

Demonstração. Seja $x, y \in X$ e $S, T \in \mathcal{B}$. Sem perda de generalidade, suponha que $C(S)(x) \leq C(S)(y)$. Assim sendo, $\rho(C(T)(x), C(T)(y))=C(T)(y) \rightarrow C(T)(x)$. Por outro lado,

$$
\begin{aligned}
C(T)(y) * C(S)(y) * C(S)(x) * I(S, T) & \leq C(T)(y) * C(S)(y) * C(S)(x) *[S(x) \rightarrow T(x)] \leq \\
& \leq C(T)(y) * S(y) * C(S)(x) * S(x) *[S(x) \rightarrow T(x)] \leq \\
& \leq C(T)(y) * R(x, y) * T(x) \leq \\
& \leq C(T)(x) .
\end{aligned}
$$

Portanto,

$$
C(S)(y) * C(S)(x) * I(S, T) \leq C(T)(y) \rightarrow C(T)(x) .
$$

Ou seja, $C$ cumpre $F \beta$. 
O exemplo a seguir mostra que a t-norma de Gödel não pode ser substituída por uma outra qualquer para que a Proposição 5.12 valha.

Exemplo 5.6. (MARTINETTI et al., 2014)Seja * uma t-norma que não a de Gödel. Assim sendo, para $a, b \in] 0,1[, a * b<a \wedge b$. Assuma que $a \leq b$. Considere o seguinte espaço de escolha fuzzy: $X=\{x, y, z\}$ e $\mathcal{B}$ a familia de conjuntos fuzzy que incluí, além do conjunto de partes de $X$ (excetuando o vazio), os conjuntos fuzzy $T_{1}$ e $T_{1}$ dados a seguir.

\begin{tabular}{|l|l|l|}
\cline { 2 - 3 } \multicolumn{1}{c|}{} & $T_{1}$ & $T_{2}$ \\
\hline$S(x)$ & 1 & 1 \\
\hline$S(y)$ & $b$ & $b$ \\
\hline$S(z)$ & $a * b$ & $a$ \\
\hline
\end{tabular}

Além disso, considere a função escolha $C$ tal que $C([x])=C([y])=C([z])=1$ e também dada pela tabela.

\begin{tabular}{|l|l|l|l|l|l|l|}
\cline { 2 - 7 } \multicolumn{1}{c|}{} & {$[x, y]$} & {$[x, z]$} & {$[y, z]$} & {$[x, y, z]$} & $T_{1}$ & $T_{2}$ \\
\hline$C(S)(x)$ & 1 & 1 & 0 & 1 & 1 & 1 \\
\hline$C(S)(y)$ & $a$ & 0 & $a$ & $a$ & $a$ & $a * b$ \\
\hline$C(S)(z)$ & 0 & 1 & 1 & 1 & $a * b$ & $a$ \\
\hline
\end{tabular}

Note que esse espaço de escolha fuzzy cumpre H1 e H2. Além disso, cumpre WFCA (as contas são omitidas). Porém,

$$
I\left(T_{1}, T_{2}\right) * C\left(T_{1}\right)(x) * C\left(T_{1}\right)(y)=1 * 1 * a>a * b=\rho\left(C\left(T_{2}\right)(x), C\left(T_{2}\right)(y)\right) .
$$

Ou seja, $C$ não cumpre $F \beta$.

Os resultados obtidos são sumarizados na Figura 5.

Por fim, são analisadas as conexões entre a regularidade de $R$, normalidade de $C$ e as condições $F \alpha, F \beta$. Antes será provado um lema útil.

Lema 5.7. Seja $I$ um conjunto de índices e $a_{i}, b_{i} \in[0,1]$ para todo $i \in I$. Então, $\bigwedge_{i \in I}\left[a_{i}\right] * \bigwedge_{i \in I}\left[b_{i}\right] \leq \bigwedge_{i \in I}\left[a_{i} * b_{i}\right]$.

Demonstração. Suponha que $\bigwedge_{i \in I}\left[a_{i}\right] * \bigwedge_{i \in I}\left[b_{i}\right]>\bigwedge_{i \in I}\left[a_{i} * b_{i}\right]$. Logo, existe um $j \in I$ tal que $\bigwedge_{i \in I}\left[a_{i}\right] * \bigwedge_{i \in I}\left[b_{i}\right]>a_{j} * b_{j}$. Mas, então,

$$
\begin{aligned}
a_{j} * b_{j} & \geq \bigwedge_{i \in I}\left[a_{i}\right] * \bigwedge_{i \in I}\left[b_{i}\right]> \\
& >a_{j} * b_{j} .
\end{aligned}
$$

Proposição 5.13. (GEORGESCU, 2007) Sejam um espaço de escolha fuzzy $(X, \mathcal{B})$ e uma função de escolha fuzzy C. Então, se $C$ é normal, $C$ cumpre $F \alpha$. 
Figura 5 - WFCA, $F \alpha$ e $F \beta$

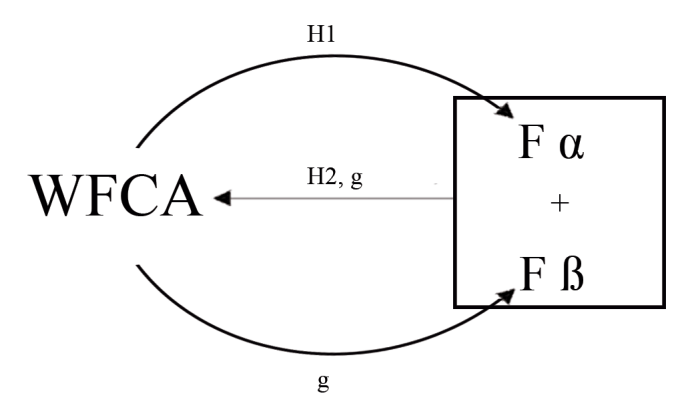

g: t-norma de Gödel

Fonte: o autor.

Demonstração. Seja $x \in X$ e $S, T \in \mathcal{B}$. Considere um $z \in X$ qualquer. Então, pela Proposição 4.3 .

$$
\begin{aligned}
S(z) *[S(z) \rightarrow T(z)] *[T(z) \rightarrow R(x, z)] & \leq T(z) *[T(z) \rightarrow R(x, z)] \leq \\
& \leq R(x, z) .
\end{aligned}
$$

Logo,

$$
[S(z) \rightarrow T(z)] *[T(z) \rightarrow R(x, z)] \leq S(z) \rightarrow R(x, z) .
$$

Como z é arbitrário,

$$
\bigwedge_{z \in X}[(S(z) \rightarrow T(z)) *(T(z) \rightarrow R(x, z))] \leq \bigwedge_{z \in X}[S(z) \rightarrow R(x, z)]
$$

Assim sendo,

$$
\begin{array}{rlrl}
I(S, T) * S(x) * C(T)(x) & =I(S, T) * S(x) * \widehat{C}(T)(x)= & & \text { normalidade } \\
& =\bigwedge_{z \in X}[S(z) \rightarrow T(z)] * S(x) * T(x) * \bigwedge_{z \in X}[T(z) \rightarrow R(x, z)] \leq & \\
& \leq S(x) * \bigwedge_{z \in X}[(S(z) \rightarrow T(z)) *(T(z) \rightarrow R(x, z))] \leq & \text { lema 5.7 } \\
& \leq S(x) * \bigwedge_{z \in X}[S(z) \rightarrow R(x, z)]= & \\
& =\widehat{C}(S)(x)=C(S)(x) . & \text { equação (5.5) }
\end{array}
$$


A proposição a seguir também aparece em Martinetti et al. (2014). No entanto, a demonstração aqui dada parece ser mais intuitiva.

Proposição 5.14. Sejam $(X, \mathcal{B})$ um espaço de escolha fuzzy e $C$ uma função de escolha fuzzy. Então, se $C$ é normal e $R$ regular, $C$ cumpre $F \beta$.

Demonstração. Sejam $x, y \in X$ e $S, T \in \mathcal{B}$. Se $C(T)(x)=C(T)(y), \rho(C(T)(x), C(T)(y))=$ 1 e não há nada para provar. Sem perda de generalidade, considere $C(T)(x)>C(T)(y)$. Lembrando que, pela Proposição 5.9, C cumpre WFCA,

$$
\begin{gathered}
C(T)(x) * I(S, T) * C(S)(x) * C(S)(y)= \\
=C(T)(x) * \bigwedge_{z \in X}[S(z) \rightarrow T(z)] * C(S)(x) * S(y) * \bigwedge_{z \in X}[S(z) \rightarrow R(y, z)] \leq \\
\leq C(T)(x) *[S(y) \rightarrow T(y)] * S(y) * S(x) *[S(x) \rightarrow R(y, x)] \leq \\
\leq C(T)(x) * T(y) * R(y, x) \leq \\
\leq C(T)(y) .
\end{gathered}
$$

Na primeira passagem utilizando a normalidade de $C$ e definição de $I(S, T)$; na segunda, o fato $C(S)(x) \leq S(x)$; na terceira a Proposição 4.3 , e na quarta o fato de $C$ cumprir WFCA. Assim sendo,

$$
\begin{aligned}
I(S, T) * C(S)(x) * C(S)(y) & \leq C(T)(x) \rightarrow C(T)(y)= \\
& =\rho(C(T)(x), C(T)(y)),
\end{aligned}
$$

uma vez que $C(T)(x)>C(T)(y)$.

Proposição 5.15. Considere * a t-norma de Gödel. Seja $m(X, \mathcal{B})$ um espaço de escolha fuzzy que cumpre H1 e H2 e C uma função de escolha fuzzy. Então, se C cumpre Fa e $F \beta, R$ é regular e $C$ é normal.

Demonstração. Pelas Proposições 5.10 e 5.7

O exemplo 5.4 também mostra que o fato da t-norma ser de Gödel é essencial para para a proposição 5.15, uma vez que, caso não fosse, pela Proposição 5.9. também não seria para a 5.10 - o que não é o caso.

A Figura 6 sintetiza os resultados obtidos. 
Figura 6 - Regularidade, normalidade, $F \alpha$ e $F \beta$

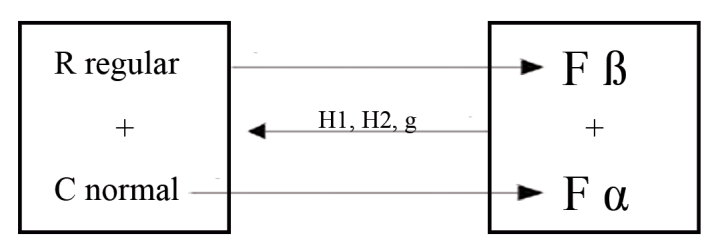

g: t-norme de Gödel

Fonte: o autor. 


\section{Conclusão}

O conhecimento sobre racionalidade possui valor prático na economia e em outras áreas que lidam com escolhas. Tal valor se dá ao buscar melhores alternativas para tentar, por um lado, descrever o comportamento racional e, por outro, implementar modelos e políticas com melhores resultados.

Nos capítulos anteriores, foi traçada a passagem dessa intuição para a formalização matemática da mesma. Para tal, buscou-se a contextualização histórica do problema, a abordagem clássica e, por fim, situações concretas que permitem uma interpretação econômica utilizando o ferramental da teoria matemática fuzzy.

Os exemplos dados no Capítulo 5 mostram que os resultados obtidos não prescindem facilmente das hipóteses utilizadas. Tendo isso em vista, o afrouxamento dos pressupostos não parece ser um caminho promissor para novos resultados. Porém, uma abordagem viável para novas conclusões pode ser a busca de uma condição que fosse suficiente, sob hipóteses mais fracas, para a validade das definições de racionalidade dadas no contexto da lógica fuzzy. 


\section{Referências}

ARROW, K. J. Rational choice functions and orderings. Econometrica, 1959.

BAETS, B. D.; MEYER, H. D. On the existence and construction of t-transitive closures. Information Sciences, 2003.

BANERJEE, A. Fuzzy choice functions, revealed preference and rationality. Fuzzy Sets and Systems, 1995.

BARROS, L. C. de; BASSANEZI, R. C.; LODWICK, W. A. A First Course in Fuzzy Logic, Fuzzy Dynamical Systems and Biomathematics - Theory and Applications. Berlin: Springer-Verlag, 2017.

CHERNOFF, H. Rational selection of decision functions. Econometrica, 1954.

GEORGESCU, I. Fuzzy Choice Functions - A Revealed Preference Approach. Berlin: Springer-Verlag, 2007.

HOUTHAKKER, H. S. Revealed preference and the utility function. Economica, 1950.

MARTINETTI, D. et al. On arrow-sen style equivalences between rationality conditions for fuzzy choice functions. Fuzzy Optimun Decision Making, 2014.

NGUYEN, H. T.; WALKER, E. A. A First Course in Fuzzy Logic. Nova Iorque: CRC Press, 1997.

ORLOVSKY, S. A. Decision-making with a fuzzy preference relation. Computing Centre of the U.S.S.R. Academy of Sciences., 1977.

RICHTER, M. K. Revealed preference theory. Econometrica, 1966.

SAMUELSON, P. A. A note on the pure theory of consumer's behavior. Economica, 1938.

SCHMITZ, F. Wittgenstein. Paris: Sociètè d'Edition Les Belles Lettres, 1999.

SEN, A. K. Quasi-transitivity, rational choice and collective decisions. Review of Economic Studies, 1969.

SEN, A. K. Choice functions and revealed preference. The Review of Economic Studies, 1971.

SEN, A. K. Social choice theory: A re-examination. Econometrica, 1977.

STOLL, R. R. Set Theory and Logic. Nova Iorque: Dover Publications, 1979.

SUZUMURA, K. Rational choice and revealed preference. The Review of Economic Studies, 1976.

ZADEH, L. A. Fuzzy sets. Information and Control, 1965. 


\section{ANEXO A - Normalidade de uma Função de Escolha}

\section{A.1 Normalidade na Teoria da Escolha Clássica}

As definições e resultados aqui apresentados estão em Suzumura (1976).

Dados um espaço de escolha $(X, \mathcal{B})$ e uma relação $Q$ em $X$, podem ser definidos os seguintes mapas com domínio em $\mathcal{B}$ e contradomínio na família de partes de $X$ :

$$
\begin{aligned}
& G_{Q}(S)=\{x \in S \mid x Q y, \forall y \in S\} \\
& M_{Q}(S)=\{x \in S \mid y Q x \Longrightarrow x Q y, \forall y \in S\}
\end{aligned}
$$

$G_{Q}(S)$ é o conjunto dos maiores elementos de $S$ segundo a relação $Q$ e $M_{Q}(S)$ o conjunto dos elementos maximais de $S$ segundo a relação $Q$. Se $Q$ é uma relação de preferência, $C$ um consumidor e $S$ um conjunto de cesta de bens, $x \in G_{Q}(S)$ indica que $x$ foi revelado ao menos tão preferido quanto todas as outas cestas em $S$. Já $x \in M_{Q}(S)$ indica que não existe cesta $y$ em $S$ que foi revelada ser ao menos tão preferida quanto a cesta $x$ sem que $x$ também tivesse sido revelada tão boa para o consumidor quanto $y$.

Uma função de escolha $C$ é dita $G$-racional ( $M$-racional) quando existe uma relação $Q$ em $X$ tal que, para todo $S \in \mathcal{B}, C(S)=G_{Q}(S)\left(C(S)=M_{Q}(S)\right)$.

Considerando a relação $R$ definida em 3.3 , uma função de escolha $C$ é $G$-normal quando, para todo $S \in \mathcal{B}, C(S)=G_{R}(S)$ e é $M$-normal quando $C(S)=M_{R}(S)$. A normalidade de uma função escolha definida em 3.5 é o mesmo que $G$-normalidade. Entretanto, o resultado abaixo mostra que essas definições são equivalentes.

Proposição A.1. Dados um espaço de escolha $(X, \mathcal{B})$ e uma função de escolha $C$, são equivalentes:

(i) Cé G-racional

(ii) Cé M-racional

(iii) Cé G-normal

(iv) $C$ é $M$-normal

Isso significa que definir a normalidade de uma função escolha $C$ como $G$-normalidade é equivalente a defini-la como $M$-normalidade, $G$-racionalidade ou $M$-racionalidade. 


\section{A.2 Normalidade na Teoria da Escolha Fuzzy}

As definições e resultados aqui apresentados estão em Martinetti et al. (2014). A intuição por trás delas é semelhante àquela do caso clássico.

Dados um espaço de escolha fuzzy $(X, \mathcal{B})$ e uma relação fuzzy $Q$ em $X$, e uma t-norma *, são definidos os seguintes mapas com domínio em $\mathcal{B}$ e contradomínio nos conjuntos fuzzy de $X$

$$
\begin{aligned}
& G_{Q}(S)(x)=S(x) * \bigwedge_{y \in X}[S(y) \rightarrow Q(x, y)] \\
& M_{Q}(S)(x)=S(x) * \bigwedge_{y \in X}[S(y) \rightarrow(Q(y, x) \rightarrow Q(x, y))]
\end{aligned}
$$

Uma função de escolha fuzzy $C$ é denominada G-racional (M-racional) quando existe uma relação fuzzy $Q$ em $X$ tal que, para todo $S \in \mathcal{B}, C(S)=G_{Q}(S)\left(C(S)=M_{Q}(S)\right)$. Levando em conta a relação fuzzy $R$ definida em 4.10, $C$ é dita $G$-normal ( $M$-normal) quando, para todo $S \in \mathcal{B}, C(S)=G_{R}(S)\left(C(S)=M_{R}(S)\right)$. A definição de normalidade dada em 4.12 equivale à de $G$-normalidade. Entretanto, o resultado abaixo mostra que, sob as hipóteses apresentadas no capítulo 5 , elas se equivalem.

Proposição A.2. Considere * uma t-norma contínua à esquerda. Dados um espaço de escolha $(X, \mathcal{B})$ que cumpra WH1 e H2 e uma função de escolha $C$, são equivalentes:

(i) Cé G-racional

(ii) $C$ é $M$-racional

(iii) $C$ é G-normal

(iv) $C$ é $M$-normal

Logo, sob as hipóteses apresentadas, definir a normalidade de $C$ como $G$-normalidade é equivalente a defini-la como $M$-normalidade, $G$-racionalidade ou $M$-racionalidade. 